\begin{tabular}{|c|l|}
\hline Title & Existence of asymmetric Burgers vortices and their asymptotic behavior at large circulations \\
\hline Author(s) & Maekawa, Y asunori \\
\hline Citation & Hokkaido University Preprint Series in Mathematics, 896, 1-34 \\
\hline Issue Date & 2008 \\
\hline DOI & 10.14943/84046 \\
\hline Doc URL & http://hdl.handle.net/2115/69705 \\
\hline Type & bulletin (article) \\
\hline File Information & pre896.pdf \\
\hline
\end{tabular}

Instructions for use 


\title{
EXISTENCE OF ASYMMETRIC BURGERS VORTICES AND THEIR ASYMPTOTIC BEHAVIOR AT LARGE CIRCULATIONS
}

\author{
YASUNORI MAEKAWA ${ }^{1}$
}

\begin{abstract}
The asymmetric Burgers vortices are vortex solutions to the three dimensional stationary Navier-Stokes equations for viscous incompressible fluids in the presence of an asymmetric background straining flow. The asymmetry of the straining flow is expressed by a non-negative parameter less than 1. The Burgers vortices have been used as a model which expresses tube-like structures of concentrated vorticity fields in turbulence, and they are numerically well investigated especially in the case of large circulation numbers. However, their existence was proved mathematically only when either the asymmetry of the straining flow is not so strong or the circulation number is sufficiently small. In this paper we prove the existence of asymmetric Burgers vortices for all circulation numbers and each asymmetry parameter less than 1 . We also obtain their asymptotic expansion at large circulation numbers, which gives an explanation for a symmetrizing effect by a fast rotation.
\end{abstract}

\section{INTRODUCTION}

In this paper we consider a stationary flow of a viscous incompressible fluid whose velocity field $U$ takes the form

$$
U\left(x_{1}, x_{2}, x_{3}\right)=u_{\lambda}\left(x_{1}, x_{2}, x_{3}\right)+u\left(x_{1}, x_{2}\right) .
$$

Here the velocity field $u_{\lambda}$ expresses an asymmetric background straining flow and is given by

$$
u_{\lambda}\left(x_{1}, x_{2}, x_{3}\right)=\left(-\frac{1+\lambda}{2} x_{1},-\frac{1-\lambda}{2} x_{2}, x_{3}\right),
$$

with a fixed parameter $\lambda \in[0,1)$. The parameter $\lambda$ represents the asymmetry of the background straining flow. The solenoidal velocity field $u$ expresses a two dimensional perturbation flow:

$$
u\left(x_{1}, x_{2}\right)=\left(u_{1}\left(x_{1}, x_{2}\right), u_{2}\left(x_{1}, x_{2}\right), 0\right), \frac{\partial u_{1}}{\partial x_{1}}+\frac{\partial u_{2}}{\partial x_{2}}=0 .
$$

We assume that the velocity field $U$ solves the stationary Navier-Stokes equations:

$$
\left\{\begin{array}{l}
-\Delta U+(U, \nabla) U+\nabla P=0, x \in \mathbb{R}^{3}, \\
\nabla \cdot U=0, x \in \mathbb{R}^{3} .
\end{array}\right.
$$

\footnotetext{
${ }^{1}$ Supported by Research Fellowships of the Japan Society for the Promotion of Science for Young Scientists.

Department of Mathematics, Hokkaido University, Sapporo, 060-0810, Japan.

E-mail: yasunori@math.sci.hokudai.ac.jp
} 
Here $\Delta=\sum_{i=1}^{3} \frac{\partial^{2}}{\partial x_{i}},(U, \nabla)=\sum_{i=1}^{3} U_{i} \frac{\partial}{\partial x_{i}}$, and $\nabla \cdot U=\sum_{i=1}^{3} \frac{\partial U_{i}}{\partial x_{i}}$. We write $\partial_{i}$ instead of $\frac{\partial}{\partial x_{i}}$ for simplicity. The function $P$ represents a pressure field of the fluid.

We are interested in the behavior of the vorticity field. Taking the rotation of $U=u_{\lambda}+u$, we see that the vorticity $\Omega=\nabla \times U$ is given by

$$
\Omega\left(x_{1}, x_{2}, x_{3}\right)=\left(0,0, \omega\left(x_{1}, x_{2}\right)\right)
$$

where $\omega=\partial_{1} u_{2}-\partial_{2} u_{1}$. If $\omega$ is smooth and integrable, and $u$ decays at infinity, then the velocity field $u$ is recovered from the vorticity field $\omega$ via the Biot-Savart law:

$$
u=K * \omega,
$$

where the convolution kernel $K$ is given by

$$
K(x)=\frac{1}{2 \pi} \frac{x^{\perp}}{|x|^{2}}, x^{\perp}=\left(-x_{2}, x_{1}\right) .
$$

The value $\int_{\mathbb{R}^{2}} \omega(x) d x$ is called the total circulation and its absolute value is called the vortex Reynolds number. Let $\alpha \in \mathbb{R}$ be a given real number. We consider the vorticity field $\omega$ whose total circulation is $\alpha$. Since $U$ satisfies (NS), we see that $\omega$ solves the following equation

$$
\left\{\begin{array}{l}
(\mathcal{L}+\lambda \mathcal{M}) \omega-B(\omega, \omega)=0, x \in \mathbb{R}^{2}, \\
\int_{\mathbb{R}^{2}} \omega(x) d x=\alpha,
\end{array}\right.
$$

where

$$
\begin{aligned}
& \mathcal{L}=\Delta+\frac{x}{2} \cdot \nabla+1, \\
& \mathcal{M}=\frac{1}{2}\left(x_{1} \partial_{1}-x_{2} \partial_{2}\right), \\
& B(f, h)=(K * f, \nabla) h .
\end{aligned}
$$

Conversely, if $\omega$ is a solution to $\left(B_{\lambda, \alpha}\right)$, then we can show that $u_{\lambda}+K * \omega$ gives a solution to (NS) with a suitably determined pressure $P$. We call solutions to $\left(B_{\lambda, \alpha}\right)$ the Burgers vortices.

Let $G$ be the two dimensional Gauss kernel:

$$
G(x)=\frac{1}{4 \pi} e^{-\frac{|x|^{2}}{4}}
$$

Then by direct calculations, we see that $G$ satisfies

$$
\mathcal{L} G=0,(K * G, \nabla) G=0 .
$$

Thus $\alpha G$ solves $\left(B_{\lambda, \alpha}\right)$ for $\lambda=0$. This exact solution was found by Burgers [1], and it is called the axisymmetric Burgers vortex. When $\lambda \in(0,1)$ a solution to $\left(B_{\lambda, \alpha}\right)$ is called the asymmetric Burgers vortex. In this case we can not expect the explicit representation as in the axisymmetric case $\lambda=0$. So the existence of solutions to $\left(B_{\lambda, \alpha}\right)$ itself is an important problem and this is the main interest in this paper.

The Burgers vortices have been used as a model of concentrated vorticity fields in turbulence. It is numerically observed that the region of intense vorticity fields in three dimensional turbulence tends to form a lot of tube-like structures, and that each vortex-tube is well described by the Burgers vortices. From this physical point of view, they have been numerically studied mainly in the case of large vortex 
Reynolds numbers, since the vortex Reynolds number is considered to represent the magnitude of the vorticity; see Robinson-Saffman [17], Kida-Ohkitani [10], MoffattKida-Ohkitani [12], Prochazka-Pullin [15], Prochazka-Pullin [16]. One of the most interesting features of their numerical results is that as the vortex Reynolds number is increasing, the Burgers vortices tend to be more circular even when the asymmetry parameter is not zero. Based on their numerical results, Robinson and Saffman conjectured in [17] that the asymmetric Burgers vortices would rigorously exist for any $\lambda \in[0,1)$ at least when $\frac{\lambda}{1+|\alpha|}$ is sufficiently small. In [12] the above property of the Burgers vortices is explained by obtaining a formal asymptotic expansion at large vortex Reynolds numbers. First mathematical approach to this problem was done by Gallay and Wayne in [6] and [7]. In [7] the existence of the Burgers vortices is proved in the Gaussian weighted $L^{2}$ space for any $|\alpha|$ if the asymmetry parameter $\lambda$ is sufficiently small $\left(\lambda<<\frac{1}{2}\right)$. This result is extended by the author [14] and it is shown that the Burgers vortices exist in the Gaussian weighted $L^{2}$ space if the parameters $\lambda$ and $\alpha$ satisfy $\lambda \in\left[0, \frac{1}{2}\right)$ and $\frac{\lambda}{1+|\alpha|} \leq \epsilon(\lambda)<<1$. Here the constant $\epsilon(\lambda)$ is taken as $\lim _{\lambda \rightarrow \frac{1}{2}} \epsilon(\lambda)=0$. Both in [7] and [14] the asymptotic expansion at large $|\alpha|$ indicated by [12] is rigorously verified. For the case $\lambda \in\left[\frac{1}{2}, 1\right)$ the problem becomes more complicated because the operator $\lambda \mathcal{M}$ breaks the symmetry of the equation strongly. As far as the author knows, the only mathematical results in this case are the results by [6] in which it is proved that the Burgers vortices exist in the polynomial weighted $L^{2}$ space when $|\alpha|$ is sufficiently small depending on $\lambda \in[0,1)$. So the above conjecture has been open especially in the case $\lambda \in\left[\frac{1}{2}, 1\right)$. The details on the difficulty in this case are explained later.

In this paper we will prove that the Burgers vortices exist for each asymmetry parameter $\lambda \in[0,1)$ and all circulation numbers $\alpha$, which gives the affirmative answer to Robinson-Saffman's question. Moreover, we verify Moffatt-Kida-Ohkitani's asymptotic expansion at large vortex Reynolds numbers ([12]) for any $\lambda \in[0,1)$. The stability of the Burgers vortices is also an important question, but we do not discuss in this paper. Instead, we give a remark on several known results and open problems about their stability in Remark 6.1.

To state our results precisely, let us introduce function spaces. Let $G_{\lambda}$ be the function defined by

$$
G_{\lambda}(x)=\frac{1-\lambda}{4 \pi} \exp \left(-\frac{1-\lambda}{4}|x|^{2}\right) .
$$

Let $X_{\lambda}, Y_{\lambda}$ be the complex Hilbert spaces defined as follows.

$$
\begin{aligned}
& X_{\lambda}=\left\{w \in L^{2}\left(\mathbb{R}^{2}\right) \mid G_{\lambda}^{-\frac{1}{2}} w \in L^{2}\left(\mathbb{R}^{2}\right), \int_{\mathbb{R}^{2}} w d x=0,\right. \\
& \left.<w_{1}, w_{2}>_{X_{\lambda}}=\int_{\mathbb{R}^{2}} G_{\lambda}^{-1}(x) w_{1}(x) \overline{w_{2}(x)} d x\right\} \\
& Y_{\lambda}=\left\{w \in X_{\lambda} \mid \partial_{i} w \in X_{\lambda}, i=1,2,\right. \\
& \left.<w_{1}, w_{2}>_{Y_{\lambda}}=\int_{\mathbb{R}^{2}} G_{\lambda}^{-1}(x)\left(w_{1}(x) \overline{w_{2}(x)}+\nabla w_{1}(x) \cdot \nabla \overline{w_{2}(x)}\right) d x\right\} .
\end{aligned}
$$

We also define the subspace of $X_{\lambda}$ 


$$
\begin{aligned}
& W_{\lambda}=\left\{w \in X_{\lambda} \mid, G_{\lambda}^{-\frac{1}{2}} x_{i} w \in L^{2}\left(\mathbb{R}^{2}\right) i=1,2\right. \\
& \left.<w_{1}, w_{2}>_{W_{\lambda}}=\int_{\mathbb{R}^{2}} G_{\lambda}^{-1}(x)\left(w_{1}(x) \overline{w_{2}(x)}+|x|^{2} w_{1}(x) \overline{w_{2}(x)}\right) d x .\right\}
\end{aligned}
$$

The space $X_{0}$ (and also $Y_{0}$ ) are used in [7], [13], and [14]. Let $\mathcal{G}_{\lambda}$ be the function given by

$$
\mathcal{G}_{\lambda}(x)=\frac{\sqrt{1-\lambda^{2}}}{4 \pi} e^{-\frac{1+\lambda}{4} x_{1}^{2}-\frac{1-\lambda}{4} x_{2}^{2}} .
$$

Note that $(\mathcal{L}+\lambda \mathcal{M}) \mathcal{G}_{\lambda}=0$ and $\int_{\mathbb{R}^{2}} \mathcal{G}_{\lambda}(x) d x=1$ hold. Our first main result is as follows.

Theorem 1.1 (Existence of asymmetric Burgers vortices for any parameters). Let $\lambda \in[0,1)$ and $\alpha \in \mathbb{R}$. Then there is a (real valued) solution $\omega_{\lambda, \alpha}$ to $\left(B_{\lambda, \alpha}\right)$ such that $\omega_{\lambda, \alpha}-\alpha \mathcal{G}_{\lambda} \in Y_{\lambda} \cap W_{\lambda}$.

The above theorem will be proved in Section 6 by a suitable application of the Schauder fixed point theorem. The key idea is to reduce $\left(B_{\lambda, \alpha}\right)$ to an evolution equation by introducing the scaling variables $x=\frac{\xi}{\sqrt{\tau}}$ and apply the results of CarlenLoss [2] in order to obtain a priori $L^{p}$ estimates for solutions. Its argument is not so complicated, but instead, we do not have detailed informations on the solutions. Especially, the method used in the proof of Theorem 1.1 is less helpful if one want to explain why the asymmetric Burgers vortex tends to be circular when the vortex Reynolds number is large. So we need a completely different approach in the study of the Burgers vortices for large $|\alpha|$.

Let $n \in \mathbb{Z}$ and let $\mathbb{P}_{n}$ be the orthogonal projection defined by

$$
\mathbb{P}_{n} w=w_{n}(r) e^{i n \theta}, \quad w_{n}(r)=\frac{1}{2 \pi} \int_{0}^{2 \pi} w(r \cos \theta, r \sin \theta) e^{-i n \theta} d \theta .
$$

We set

$$
\begin{aligned}
& \mathbb{P}_{n} X_{\lambda}=\left\{\mathbb{P}_{n} w \mid w \in X_{\lambda}\right\}, \\
& \mathbb{P}^{e} X_{\lambda}=\oplus_{n \in \mathbb{Z}} \mathbb{P}_{2 n} X_{\lambda} .
\end{aligned}
$$

It will be useful to define the subspace of all "non-radially" symmetric functions:

$$
\mathbb{P}_{0}^{\perp} X_{\lambda}=\left\{\mathbb{P}_{0}^{\perp} w \mid w \in X_{\lambda}, \mathbb{P}_{0}^{\perp}=I-\mathbb{P}_{0}\right\} .
$$

For a given $h \in Y_{\lambda}$ we define an integro-differential operator $\Lambda_{h}$ on $Y_{\lambda}$ as

$$
\Lambda_{h} f=B(h, f)+B(f, h) .
$$

In Section 2 we will see that $\mathbb{P}^{e} X_{\lambda}$ is invariant under the action of $\Lambda_{h}$ if $h$ belongs to $\mathbb{P}^{e} X_{\lambda}$. Let $w_{\infty} \in Y_{0} \cap W_{0}$ be the function which satisfies the equation

$$
\mathcal{M} G=\Lambda_{G} w_{\infty} .
$$

The existence of $w_{\infty}$ is proved in [7]; see also [12]. Especially, $w_{\infty}$ is uniquely determined in $\mathbb{P}_{-2} X_{\lambda} \cup \mathbb{P}_{2} X_{\lambda}$.

Our second result is the existence and the asymptotic behavior of the Burgers vortices for large vortex Reynolds numbers. 
Theorem 1.2 (Existence and asymptotics behavior at large circulations). Let $\lambda \in$ $[0,1)$. Then there is a positive number $\Theta_{1}=\Theta_{1}(\lambda) \geq 0$ such that for any $\alpha \in \mathbb{R}$ with $|\alpha| \geq \Theta_{1}$ there exists a (real valued) solution $\omega_{\lambda, \alpha}$ of $\left(B_{\lambda, \alpha}\right)$ satisfying $\omega_{\lambda, \alpha}-\alpha G \in$ $\mathbb{P}^{e} X_{\lambda}$ and

$$
\left\|\omega_{\lambda, \alpha}-\alpha G-\lambda w_{\infty}\right\|_{Y_{\lambda} \cap W_{\lambda}} \leq \frac{\lambda M(\lambda)}{1+|\alpha|}
$$

where the constant $M(\lambda)$ depends only on $\lambda$. The constants $\Theta_{1}(\lambda)$ and $M(\lambda)$ are taken as

$$
\lim _{\lambda \rightarrow 1} \Theta_{1}(\lambda)=\lim _{\lambda \rightarrow 1} M(\lambda)=\infty .
$$

When $|\alpha|$ is large, we also have the uniqueness around $\alpha G+\lambda w_{\infty}$ as follows.

Theorem 1.3 (Uniqueness at large circulations). Let $\lambda \in[0,1)$. Then for any $\tau>0$ there is a positive number $\Theta_{2}=\Theta_{2}(\lambda, \tau) \geq \Theta_{1}$ such that for any $\alpha$ with $|\alpha| \geq \Theta_{2}$, there exists at most one solution of $\left(B_{\lambda, \alpha}\right)$ in the ball

$$
\mathcal{B}_{\tau}=\left\{f \in L^{2}\left(\mathbb{R}^{2}\right) \mid f-\alpha G \in \mathbb{P}^{e} X_{\lambda},\left\|f-\alpha G-\lambda w_{\infty}\right\|_{Y_{\lambda} \cap W_{\lambda}} \leq \tau\right\} .
$$

For each $\lambda \in[0,1)$ the constant $\Theta_{2}(\lambda, \tau)$ is taken as

$$
\lim _{\tau \rightarrow \infty} \Theta_{2}(\lambda, \tau)=\infty .
$$

Remark 1.1 (Large-Reynolds-number asymptotics). As stated previously, Moffatt, Kida, and Ohkitani indicated in [12] that the asymmetric Burgers vortex would be expanded around $\alpha G+\lambda w_{\infty}$ when $\frac{\lambda}{1+|\alpha|}$ is sufficiently small for any $\lambda \geq 0$. This expansion was rigorously recovered by Gallay and Wayne in [7] when $\lambda$ is sufficiently small, and then by [14] when $\lambda<\frac{1}{2}$. Theorem 1.2 shows that for any $\lambda \in[0,1)$ there is a solution which satisfies the above expansion. Unfortunately, we do not know whether or not the solution constructed in Theorem 1.1 satisfies (1.19) and coincides with the solution obtained in Theorem 1.2.

In order to prove Theorem 1.2 and Theorem 1.3, we first expand $\left(B_{\lambda, \alpha}\right)$ around $\alpha G+\lambda w_{\infty}$. Then we get the equation for $w=\omega-\alpha G-\lambda w_{\infty}$ :

$$
\left(\mathcal{L}+\lambda \mathcal{M}-\alpha \Lambda_{G}\right) w=B(w, w)+\lambda \Lambda_{w_{\infty}} w+\lambda f_{\lambda} .
$$

The function $f_{\lambda}$ is defined as

$$
f_{\lambda}=-\mathcal{L} w_{\infty}+\lambda\left(B\left(w_{\infty}, w_{\infty}\right)-\mathcal{M} w_{\infty}\right) .
$$

It is known that $f_{\lambda} \in Y_{0} \cap W_{0} \cap \mathbb{P}^{e} X_{0}$ and $\mathbb{P}_{0} f_{\lambda}=0$; see [14, Corollary 2.3]. In next section we will see that $\mathbb{P}^{e} X_{\lambda}$ is invariant under the equations (1.22).

Since the operator $\Lambda_{G}$ with the domain $D\left(\Lambda_{G}\right)=Y_{\lambda}$ is not a closed operator (but closable) in $X_{\lambda}$, we consider the closure of $\Lambda_{G}$ in $X_{\lambda}$ instead of $\Lambda_{G}$ itself. We set

$$
\Lambda=\overline{\Lambda_{G}} ; \text { the closure of } \Lambda_{G} \text { in } X_{\lambda} .
$$

In fact, the operator $\Lambda$ is given in terms of polar coordinates and the Fourier series expansion with respect to the angular variable; see Section 2 for details.

Let us state the difficulty of this problem and rough idea to overcome it. The most important step to solve (1.22) is to consider the linearized problem

$$
\mathcal{L}_{\lambda, \alpha} w:=(\mathcal{L}+\lambda \mathcal{M}-\alpha \Lambda) w=f .
$$


The main difficulty comes from the operator $\lambda \mathcal{M}$, since it leads to a slow spatial decay in $x_{2}$ direction and also breaks the symmetry of the equation. If $\lambda<\frac{1}{2}$, we can find solutions of (1.25) in the Gaussian weighted $L^{2}$ space $X_{0}\left(X_{\lambda}\right.$ for $\left.\lambda=0\right)$ at least for large $|\alpha|$; see [7] and [14]. The only reason we can treat the equation (1.25) even for large $|\alpha|$ in $X_{0}$ is that $\Lambda$ is skew-symmetric in $X_{0}$ which is discovered by Gallay and Wayne in [5]. The skew-symmetry of $\Lambda$ enables us to give uniform (or better) estimates for linearized operators $(\mathcal{L}-\alpha \Lambda)^{-1}$ or $(\mathcal{L}+\lambda \mathcal{M}-\alpha \Lambda)^{-1}$ at large $|\alpha|$. To explain this, let us recall the argument used in [5] or [7]; see also [13], [14]. Let $h \in X_{0}$ be the solution of the equation $(\mathcal{L}-\alpha \Lambda) h=f$ for $f \in X_{0}$. Then we have

$$
\begin{aligned}
\operatorname{Re}<f, h>_{X_{0}} & =\operatorname{Re}<(\mathcal{L}-\alpha \Lambda) h, h>_{X_{0}} \\
& =-\operatorname{Re}<(-\mathcal{L}) h, h>_{X_{0}} \\
& =-\left\|(-\mathcal{L})^{\frac{1}{2}} h\right\|_{X_{0}}^{2} \\
& \leq-\frac{1}{2}\|h\|_{X_{0}}^{2},
\end{aligned}
$$

by the skew-symmetry of $\Lambda$ in $X_{0}$. Thus we have

$$
\|h\|_{X_{0}} \leq 2\|f\|_{X_{0}}
$$

which gives the uniform estimate for $h=(\mathcal{L}-\alpha \Lambda)^{-1} f$. In fact, it seems to be quite difficult to obtain this uniform estimate directly without using the skew-symmetry of $\Lambda$.

If $\lambda \geq \frac{1}{2}$, we can no longer expect that solutions belong to $X_{0}$, because of the loss of a spatial decay by the operator $\lambda \mathcal{M}$. So we are forced to deal with the equations (1.25) in other function spaces which allow functions with slower spatial decays. However, in general, the operator $\Lambda$ is not skew-symmetric in such spaces. This causes serious difficulties to establish uniform estimates for solutions of the linearized problem with respect to $\alpha$. Especially, we need to control the term $\alpha \Lambda$ without the skew-symmetry of $\Lambda$ itself.

To overcome this difficulty, we look for a linear operator which makes $\Lambda$ skewsymmetric by its right action.

Definition 1.1 (Definition of a right skew-symmetrizer). Let $X$ be a Hilbert space and $A$ be a linear operator in $X, D(A) \subset X$. Then we call a linear operator $T$ in $X$ a right skew-symmetrizer of $A$ if the operator $A T, D(A T)=\{f \in D(T) \mid T f \in D(A)\}$ is skew-symmetric, i.e.,

$$
<A T f, h>_{X}+<f, A T h>_{X}=0
$$

for $f, h \in D(A T)$. We say $A$ is right skew-symmetrizable in $X$ if there is a right skew-symmetrizer $T$ of $A$ in $X$.

Then the following lemma is essential.

Lemma 1.1. There is a right skew-symmetrizer $T$ of the operator $\Lambda$ in $\mathbb{P}^{e} X_{\lambda}$. Moreover, $T$ satisfies the following:

(1) $T-I$ is compact in $\mathbb{P}^{e} X_{\lambda}$,

(2) $T$ is injective in $\mathbb{P}^{e} X_{\lambda}$, 
By the Fredholm alternative theorem, $T$ has the bounded inverse on $\mathbb{P}^{e} X_{\lambda}$. So we consider $v=T^{-1} w$ instead of the solution $w$ of (1.25) itself. From the relations

$$
\begin{aligned}
(\mathcal{L}+\lambda \mathcal{M}) w & =(\mathcal{L}+\lambda \mathcal{M}) v+(\mathcal{L}+\lambda \mathcal{M})(T-I) v \\
\alpha \Lambda w & =\alpha \Lambda T v
\end{aligned}
$$

we obtain the equation for $v$ :

$$
(\mathcal{L}+\lambda \mathcal{M}-\alpha \Lambda T) v=-(\mathcal{L}+\lambda \mathcal{M})(T-I) v+f .
$$

By the skew-symmetry of $\Lambda T$ and the characterization of Ker $\Lambda T$, we can show that the linear problem $(\mathcal{L}+\lambda \mathcal{M}-\alpha \Lambda T) v=f$ is uniquely solvable in $\mathbb{P}^{e} X_{\lambda}$ if $|\alpha|$ is sufficiently large. The term $-(\mathcal{L}+\lambda \mathcal{M})(T-I) v$ can be regarded as lower order, since $T-I$ is compact. Using these facts, we can show that the equation (1.27) is uniquely solvable in $\mathbb{P}^{e} X_{\lambda}$ and so is true for (1.25) by the relation $w=T v$. Then the nonlinear problem (1.22) will be solved by perturbation arguments as in [14].

To solve (1.27) we need to investigate the linear operator $\mathcal{L}+\lambda \mathcal{M}-\alpha \Lambda T$. For this purpose, we decompose $\mathcal{L}$ as

$$
\mathcal{L}=\mathcal{L}_{\lambda}+\lambda \mathcal{N}
$$

where

$$
\mathcal{L}_{\lambda}=\Delta+\frac{1-\lambda}{2} x \cdot \nabla+1-\lambda, \quad \mathcal{N}=\frac{x}{2} \cdot \nabla+1
$$

The reason why we decompose $\mathcal{L}$ as above is that the operator $\mathcal{L}_{\lambda}$ is self-adjoint in $X_{\lambda}$ with the spectrum $\sigma\left(\mathcal{L}_{\lambda}\right)=\left\{-\frac{(1-\lambda) n}{2} \mid n=1,2, \cdots\right\}$ and that both $\mathcal{L}_{\lambda}$ and $\mathcal{N}$ map $\mathbb{P}_{n} X_{\lambda} \cap D\left(\mathcal{L}_{\lambda}\right)$ to $\mathbb{P}_{n} X_{\lambda}$. Especially, we can see by direct calculations that each of $\mathcal{L}_{\lambda}, \mathcal{M}$, and $\mathcal{N}$ maps $\mathbb{P}^{e} X_{\lambda} \cap D\left(\mathcal{L}_{\lambda}\right)$ to $\mathbb{P}^{e} X_{\lambda}$.

In order to derive better properties of $\mathcal{L}_{\lambda, \alpha}$ or $\mathcal{L}+\lambda \mathcal{M}-\alpha \Lambda T$ for large $|\alpha|$, it is important to characterize the kernel of $\Lambda$ or $\Lambda T$. By a simple observation, it turns out that the kernel of $\Lambda$ or $\Lambda T$ in $\mathbb{P}^{e} X_{\lambda}$ coincides with the subspace consisting of all radially symmetric functions in $X_{\lambda}$, i.e.,

$$
\operatorname{Ker} \Lambda=\operatorname{Ker} \Lambda T=\mathbb{P}_{0} X_{\lambda} .
$$

This is useful and essential in our proof, since the decomposition of solutions into radially symmetric parts and non-radially symmetric parts matches the structure of the symmetry-breaking term $\lambda \mathcal{M} v$ or the nonlinear term $B(v, v)$. For example, if $v$ is radially symmetric, then $\mathcal{M} v$ belongs to $\mathbb{P}_{0}^{\perp} X_{\lambda}$ and $B(v, v)=0$.

We will show that $\mathcal{L}_{\lambda, \alpha}$ is invertible for large $\alpha$ and its inverse has better estimates as $|\alpha|$ is increasing. More precisely, by similar arguments as in [14], the operator norms of $\mathcal{L}_{\lambda, \alpha}^{-1} \mathbb{P}_{0}^{\perp}$ and $\mathbb{P}_{0}^{\perp} \mathcal{L}_{\lambda, \alpha}^{-1}$ are estimated as small for large $|\alpha|$, where $\mathbb{P}_{0}^{\perp}=I-\mathbb{P}_{0}$. We construct a solution to (1.22) by decomposing it into the radially symmetric part $\left(\mathbb{P}_{0} X_{\lambda}\right)$ and the non-radially symmetric part $\left(\mathbb{P}_{0}^{\perp} X_{\lambda}\right)$. Unfortunately, we do not have better estimates for $\mathbb{P}_{0} \mathcal{L}_{\lambda, \alpha}^{-1} \mathbb{P}_{0}$ even if $|\alpha|$ is large. But since the radially symmetric part of solutions to (1.22) is essentially expressed by the non-radially symmetric part of them, we can establish necessary a priori estimates for solutions to (1.22) when the vortex Reynolds number $|\alpha|$ is sufficiently large.

This paper is organized as follows. In Section 2 we summarize the properties of the operator $\mathcal{L}_{\lambda}$ and give the representation of $\Lambda$ in polar coordinates. We also recall 
the properties of the bilinear form $B(f, h)$. In Section 3 we prove that the existence of a right skew-symmetrizer $T$ of $\Lambda$ and establish some estimates for the operator $T-I$. In Section 4 we study the linearized operator $\mathcal{L}_{\lambda, \alpha}$ and prove several estimates depending on the value of $|\alpha|$. In Section 5 we construct a solution of (1.22) by the Schauder fixed point theorem. After that, the uniqueness of solutions to (1.22) is proved under the assumption of Theorem 1.3. The asymptotic expansion at large vortex Reynolds number (1.19) is also established by similar arguments as in [7] or [14]. Finally, Theorem 1.1 is proved in Section 6.

\section{Preliminaries}

2.1. Properties of $\mathcal{L}_{\lambda}$. It is well-known that the operator $\mathcal{L}_{\lambda}$ with the domain $\left\{f \in X_{\lambda} \mid \Delta f+\frac{1-\lambda}{2} x \cdot \nabla f \in X_{\lambda}\right\}$ is self-adjoint in $X_{\lambda}$; see [7, Proposition 2.1] when $\lambda=0$. The spectrum of $\mathcal{L}_{\lambda}$ consists of all eigenvalues and is given by $\left\{-\frac{(1-\lambda) n}{2} \mid n=1,2, \cdots\right\}$. The associated eigenfunctions for $-\frac{(1-\lambda) n}{2}$ are the Hermite functions $\left\{\partial_{1}^{a_{1}} \partial_{2}^{a_{2}} G_{\lambda}\right\}$ with $a_{1}+a_{2}=n$. We set

$$
X_{\lambda, 1}=\left\{f \in X_{\lambda} \mid \int_{\mathbb{R}^{2}} x_{i} f(x) d x=0, i=1,2\right\} .
$$

The space $X_{\lambda, 1}$ is nothing but the orthogonal compliment of the eigenspace $\left\{\beta_{1} \partial_{1} G_{\lambda}+\beta_{2} \partial_{2} G_{\lambda} \mid \beta_{i} \in \mathbb{C}\right\}$ in $X_{\lambda}$. The following proposition gives fundamental estimates for the inverse of $\mathcal{L}_{\lambda}$ in $X_{\lambda, 1}$. These estimates will be freely used throughout this paper.

Proposition 2.1. Let $\lambda \in[0,1)$. Then we have for any $f \in X_{\lambda, 1}$,

$$
\begin{aligned}
\left\|\left(-\mathcal{L}_{\lambda}\right)^{-\frac{1}{2}} f\right\|_{X_{\lambda}} & \leq(1-\lambda)^{-\frac{1}{2}}\|f\|_{X_{\lambda}}, \\
\|\| x \mid\left(-\mathcal{L}_{\lambda}\right)^{-\frac{1}{2}} f \|_{X_{\lambda}} & \leq \frac{2 \sqrt{6}}{1-\lambda}\|f\|_{X_{\lambda}}, \\
\left\|\nabla\left(-\mathcal{L}_{\lambda}\right)^{-\frac{1}{2}} f\right\|_{X_{\lambda}} & \leq \sqrt{2}\|f\|_{X_{\lambda}}, \\
\left\|\left(-\mathcal{L}_{\lambda}\right)^{-\frac{1}{2}} \partial_{i} f\right\|_{X_{\lambda}} & \leq(\sqrt{2}+\sqrt{6})\|f\|_{X_{\lambda}}, i=1,2, \\
\left\|\left(-\mathcal{L}_{\lambda}\right)^{-\frac{1}{2}} x_{i} f\right\|_{X_{\lambda}} & \leq \frac{2 \sqrt{6}}{1-\lambda}\|f\|_{X_{\lambda}}, i=1,2 .
\end{aligned}
$$

In particular, the operator $\left(-\mathcal{L}_{\lambda}\right)^{-\frac{1}{2}}$ is compact in $X_{\lambda, 1}$.

Proof. When $\lambda=0$ the above estimates (2.2), (2.3), (2.4), and (2.6) are proved in [7, Proposition 2.1], and the estimate (2.5) is obtained in the proof of [14, Proposition 2.1]. Although the estimates for $\lambda>0$ can be obtained by the same arguments as in these papers, we give the proof here for convenience to readers.

Since $-\mathcal{L}_{\lambda} \geq 1-\lambda$ in $X_{\lambda, 1}$, we have

$$
\left\|\left(-\mathcal{L}_{\lambda}\right)^{\frac{1}{2}} h\right\|_{X_{\lambda}}^{2}=<\left(-\mathcal{L}_{\lambda}\right) h, h>_{X_{\lambda}} \geq(1-\lambda)\|h\|_{X_{\lambda}}^{2},
$$

thus

$$
\left\|\left(-\mathcal{L}_{\lambda}\right)^{-\frac{1}{2}} f\right\|_{X_{\lambda}} \leq(1-\lambda)^{-\frac{1}{2}}\|f\|_{X_{\lambda}}
$$

for $f \in X_{\lambda, 1}$.

By direct calculations we observe that 


$$
\begin{aligned}
<\left(-\mathcal{L}_{\lambda}\right) h, h>_{X_{\lambda}} & =\int_{\mathbb{R}^{2}}\left|\nabla\left(G_{\lambda}^{-\frac{1}{2}} h\right)(x)\right|^{2} d x+\frac{(1-\lambda)^{2}}{16} \int_{\mathbb{R}^{2}} G_{\lambda}^{-1}|x|^{2}|h(x)|^{2} d x \\
& -\frac{1-\lambda}{2} \int_{\mathbb{R}^{2}} G_{\lambda}^{-1}|h(x)|^{2} d x \\
& \geq \frac{(1-\lambda)^{2}}{16}|| x \mid h\left\|_{X_{\lambda}}^{2}-\frac{1-\lambda}{2}\right\| h \|_{X_{\lambda}}^{2} .
\end{aligned}
$$

Hence we have

$$
\frac{(1-\lambda)^{2}}{16}\left|\left\|x \mid\left(-\mathcal{L}_{\lambda}\right)^{-\frac{1}{2}} f\right\|_{X_{\lambda}}^{2} \leq\|f\|_{X_{\lambda}}^{2}+\frac{1-\lambda}{2}\left\|\left(-\mathcal{L}_{\lambda}\right)^{-\frac{1}{2}} f\right\|_{X_{\lambda}}^{2} \leq \frac{3}{2}\|f\|_{X_{\lambda}}^{2}\right.
$$

that is,

$$
\left\||| x \mid\left(-\mathcal{L}_{\lambda}\right)^{-\frac{1}{2}} f\right\|_{X_{\lambda}} \leq \frac{2 \sqrt{6}}{1-\lambda}\|f\|_{X_{\lambda}} .
$$

We also have the equality

$$
<\left(-\mathcal{L}_{\lambda}\right) h, h>_{X_{\lambda}}=\|\nabla h\|_{X_{\lambda}}^{2}-(1-\lambda)\|h\|_{X_{\lambda}}^{2} .
$$

So we have

$$
\left\|\nabla\left(-\mathcal{L}_{\lambda}\right)^{-\frac{1}{2}} f\right\|_{X_{\lambda}}^{2}=\|f\|_{X_{\lambda}}^{2}+(1-\lambda)\left\|\left(-\mathcal{L}_{\lambda}\right)^{-\frac{1}{2}} f\right\|_{X_{\lambda}}^{2} \leq 2\|f\|_{X_{\lambda}}^{2},
$$

thus,

$$
\left\|\nabla\left(-\mathcal{L}_{\lambda}\right)^{-\frac{1}{2}} f\right\|_{X_{\lambda}} \leq \sqrt{2}\|f\|_{X_{\lambda}}
$$

The estimate (2.5) is proved by the duality argument. Indeed, from the equality $<\left(-\mathcal{L}_{\lambda}\right)^{-\frac{1}{2}} \partial_{i} f, h>_{X_{\lambda}}=-\frac{1-\lambda}{2}<f, x_{i}\left(-\mathcal{L}_{\lambda}\right)^{-\frac{1}{2}} h>_{X_{\lambda}}-<f, \partial_{i}\left(-\mathcal{L}_{\lambda}\right)^{-\frac{1}{2}} h>_{X_{\lambda}}$ for any $h \in X_{\lambda}$, we have by the estimates (2.3) and (2.4),

$$
\left|<\left(-\mathcal{L}_{\lambda}\right)^{-\frac{1}{2}} \partial_{i} f, h>_{X_{\lambda}}\right| \leq \sqrt{6}\|f\|_{X_{\lambda}}|| h\left\|_{X_{\lambda}}+\sqrt{2}\right\| f\left\|_{X_{\lambda}}\right\| h \|_{X_{\lambda}} .
$$

This gives (2.5) by the duality. The estimate (2.6) is obtained similarly by (2.3) and by the equality

$$
<\left(-\mathcal{L}_{\lambda}\right)^{-\frac{1}{2}} x_{i} f, h>_{X_{\lambda}}=<f, x_{i}\left(-\mathcal{L}_{\lambda}\right)^{-\frac{1}{2}} h>_{X_{\lambda}} .
$$

We omit the details. The proof of the proposition is complete.

2.2. Representation of $\Lambda$. In this section we consider the operator $\Lambda$. We recall that $\Lambda$ is represented in terms of polar coordinates as follows; see [5, Section 4.1.1].

Let $w \in X_{\lambda}$ and $\mathbb{P}_{n} w=w_{n}(r) e^{i n \theta}$.

Then we see that $w_{n}$ belongs to the Hilbert space

$$
\begin{aligned}
& Z_{\lambda}=\left\{f:\left.\mathbb{R}_{+} \rightarrow \mathbb{C}\left|\int_{0}^{\infty} r g_{\lambda}^{-1}(r)\right| f(r)\right|^{2} d r<\infty\right\}, \\
&<f, h>_{Z_{\lambda}}=\int_{0}^{\infty} r g_{\lambda}^{-1}(r) f(r) \overline{h(r)} d r .
\end{aligned}
$$


Here

$$
g_{\lambda}(r)=\frac{1-\lambda}{4 \pi} e^{-\frac{1-\lambda}{4} r^{2}} .
$$

For simplicity, we write $g(r)$ instead of $g_{0}(r)$.

Let $\Lambda_{a, n}, \Lambda_{b, n}$ be the linear operators on $Z_{\lambda}$ defined by $\Lambda_{a, 0}=\Lambda_{b .0}=0$ and

$$
\begin{aligned}
& \Lambda_{a, n}(f)=i n \varphi f, \\
& \Lambda_{b, n}(f)=-i n g \Omega_{n}(f),
\end{aligned}
$$

where

$$
\begin{aligned}
& \varphi(r)=\frac{1}{2 \pi r^{2}}\left(1-e^{-\frac{r^{2}}{4}}\right), \\
& \Omega_{n}(f)(r)=\frac{1}{4|n|}\left(\int_{0}^{r}\left(\frac{s}{r}\right)^{|n|} s f(s) d s+\int_{r}^{\infty}\left(\frac{r}{s}\right)^{|n|} s f(s) d s\right) .
\end{aligned}
$$

It is not difficult to see that for each $n \in \mathbb{Z}$ the operators $\Lambda_{a, n}$ and $\Lambda_{b, n}$ are bounded in $Z_{\lambda}$. We set

$$
\begin{aligned}
& \Lambda_{a} w=\sum_{n \in \mathbb{Z}} \Lambda_{a, n}\left(w_{n}\right) e^{i n \theta}, D\left(\Lambda_{a}\right)=\left\{w \in X_{\lambda} \mid \sum_{n \in \mathbb{Z}} n^{2}\left\|\varphi w_{n}\right\|_{X_{\lambda}}^{2}<\infty\right\}, \\
& \Lambda_{b} w=\sum_{n \in \mathbb{Z}} \Lambda_{b, n}\left(w_{n}\right) e^{i n \theta}, D\left(\Lambda_{b}\right)=X_{\lambda} .
\end{aligned}
$$

Indeed, we can check that $\Lambda_{b}$ is bounded in $X_{\lambda}$ by using the Hölder inequality. Then the operator $\Lambda$ is expressed by

$$
\Lambda w=\Lambda_{a} w+\Lambda_{b} w .
$$

We note that all of $\Lambda, \Lambda_{a}$, and $\Lambda_{b}$ map $\mathbb{P}_{n} X_{\lambda}$ into $\mathbb{P}_{n} X_{\lambda}$.

The following proposition gives the characterization of the kernel of $\Lambda$ or $\Lambda_{a}$.

Proposition 2.2. Let $\Lambda, \Lambda_{a}$ be the operators given as above. Then we have

$$
\begin{aligned}
\operatorname{Ker} \Lambda & =\mathbb{P}_{0} X_{\lambda} \oplus\left\{\beta_{1} \partial_{1} G+\beta_{2} \partial_{2} G \mid \beta_{i} \in \mathbb{R}\right\}, \\
\operatorname{Ker} \Lambda_{a} & =\mathbb{P}_{0} X_{\lambda} .
\end{aligned}
$$

Proof. When $\lambda=0$, the characterization (2.21) is already obtained by [13, Lemma 1.2]. We easily see that the same argument can be applied and (2.21) is true for any $\lambda \in(0,1)$. We omit the details. As for $(2.22)$, we note that the function $\varphi$ is strictly positive. Thus $\Lambda_{a, n} f \equiv 0$ if and only if $f \equiv 0$ when $|n| \geq 1$, which gives (2.22).

The above characterizations will play important roles when we show the existence of a right skew-symmetrizer of $\Lambda$ and construct solutions to (1.22).

2.3. Estimates for the bilinear form $B(f, h)$. In this section we consider the bilinear form $B(f, h)=(K * f, \nabla) h$ which plays essential roles in our paper. First we recall the following proposition. Let $\mathbb{P}_{0}^{\perp}=I-\mathbb{P}_{0}$.

Proposition 2.3. Let $f \in \mathbb{P}_{n} X_{\lambda}$ and $h \in \mathbb{P}_{m} X_{\lambda} \cap Y_{\lambda}$. Then $B(f, h) \in \mathbb{P}_{n+m} X_{\lambda}$. Especially, if $f \in \mathbb{P}_{0} X_{\lambda}$ or $h \in \mathbb{P}_{0} X_{\lambda} \cap Y_{\lambda}$, then $B(f, h) \in \mathbb{P}_{0}^{\perp} X_{\lambda}$. 
Proof. The proof is the same as in [14, Proposition 2.2], so we omit it.

As a simple corollary of this proposition, we see that $\mathbb{P}^{e} X_{\lambda}$ is invariant under the action of $B(f, h)$ as follows.

Corollary 2.1. If $f \in \mathbb{P}^{e} X_{\lambda}$ and $h \in \mathbb{P}^{e} X_{\lambda} \cap Y$, then $B(f, h) \in \mathbb{P}^{e} X_{\lambda}$.

Proof. Since $\mathbb{P}^{e} X_{\lambda}=\oplus_{n \in \mathbb{Z}} \mathbb{P}_{2 n} X_{\lambda}$ the assertion is a direct consequence of Proposition 2.3 .

Remark 2.1. Note that $w_{\infty} \in \mathbb{P}^{e} X_{\lambda}$ and the closed subspace $\mathbb{P}^{e} X_{\lambda}$ is invariant under the action of the operators $\mathcal{L}_{\lambda}, \mathcal{N}$, and $\mathcal{M}$. Thus from the the above corollary and the representations of $\Lambda_{a}$ and $\Lambda_{b}$, we see that $\mathbb{P}^{e} X_{\lambda}$ is invariant under the equation (1.22).

We set $A_{\lambda}=\left(-\mathcal{L}_{\lambda}\right)^{\frac{1}{2}}$. For $\gamma \in[0,1]$ we set $\|f\|_{D\left(A_{\lambda}^{\gamma}\right)}=\|f\|_{X_{\lambda}}+\left\|A_{\lambda}^{\gamma} f\right\|_{X_{\lambda}}$ (the graph norm). Note that this is equivalent with $\left\|A_{\lambda}^{\gamma} f\right\|_{X_{\lambda}}$ since $A_{\lambda}$ has a bounded inverse defined on $X_{\lambda}$, and that $\|\cdot\|_{D\left(A_{\lambda}\right)}$ is equivalent with $\|\cdot\|_{Y_{\lambda} \cap W_{\lambda}}$ by the estimates (2.8) and (2.10).

Proposition 2.4. Let $\gamma \in(0,1]$. Let $f \in D\left(A_{\lambda}^{\gamma}\right)$ and $h \in X_{\lambda}$. Then we have

$$
\left\|\left(-\mathcal{L}_{\lambda}\right)^{-\frac{1}{2}} B(f, h)\right\|_{X_{\lambda}} \leq C\|f\|_{D\left(A_{\lambda}^{\gamma}\right)}\|h\|_{X_{\lambda}} .
$$

Here the constant $C$ depends only on $\lambda$ and $\gamma$.

Proof. First of all we note that $A_{\lambda}$ is sectorial by [4, Corollary II.4.7], so we can use the interpolation arguments in [11]. When $\lambda=0$, the above estimate is obtained in Section 3. We recall the argument there. For $\gamma \in(0,1)$ we fix $\sigma \in(0, \gamma)$. Let $r \in(2,3)$ be such that $\sigma=1-\frac{2}{r}$. By the equality $B(f, h)=\nabla \cdot(h K * f)$ and the inequality (2.5), we have

$$
\begin{aligned}
\left\|\left(-\mathcal{L}_{\lambda}\right)^{-\frac{1}{2}} B(f, h)\right\|_{X_{\lambda}} & \leq C\|h K * f\|_{X_{\lambda}} \\
& \leq C\|K * f\|_{L^{\infty}}\|h\|_{X_{\lambda}} \\
& \leq C\|K * f\|_{L^{q}}^{\frac{1}{4}}\|\nabla K * f\|_{L^{r}}^{\frac{3}{4}}\|h\|_{X_{\lambda}},
\end{aligned}
$$

where $C$ depends only on $r$. In the last line we used the Gagliardo-Nirenberg inequality for $r \in(2,3)$ and $\frac{1}{3 q}=\frac{1}{2}-\frac{1}{r}$. Note that $q \in(2, \infty)$. By the HardyLittlewood-Sobolev inequality we have

$$
\|K * f\|_{L^{q}} \leq C\|f\|_{L^{p}}
$$

for $\frac{1}{p}=\frac{1}{q}+\frac{1}{2}$. Since $p \in(1,2)$ we see from the Hölder inequality,

$$
\|K * f\|_{L^{q}} \leq C\left\|G_{\lambda}^{\frac{1}{2}}\right\|_{L^{q}}\left\|G_{\lambda}^{-\frac{1}{2}} f\right\|_{L^{2}}=C(1-\lambda)^{\frac{1}{2}-\frac{1}{q}}\|f\|_{X_{\lambda}}
$$

On the other hand, we have from the Calderón-Zygmund inequality,

$$
\|\nabla K * f\|_{L^{r}} \leq C\|f\|_{L^{r}} .
$$

Collecting these, we obtain

$$
\left\|\left(-\mathcal{L}_{\lambda}\right)^{-\frac{1}{2}} B(f, h)\right\|_{X_{\lambda}} \leq C(1-\lambda)^{\frac{1}{4}\left(\frac{1}{2}-\frac{1}{q}\right)}\|f\|_{X_{\lambda}}^{\frac{1}{4}}\|f\|_{L^{r}}^{\frac{3}{4}}\|h\|_{X_{\lambda}} .
$$


Next we observe that

$$
\|f\|_{L^{r}} \leq C\|f\|_{L^{2}}^{1-\sigma}\|\nabla f\|_{L^{2}}^{\sigma},
$$

for $\sigma=1-\frac{2}{r}$ by the Gagliardo-Nirenberg inequality. Hence by (2.4), the estimate

$$
\|f\|_{L^{r}} \leq C(1-\lambda)^{\frac{1}{2}}\|f\|_{X_{\lambda}}^{1-\sigma}\left\|A_{\lambda} f\right\|_{X_{\lambda}}^{\sigma}
$$

holds, which shows that by the interpolation argument

$$
\|f\|_{L^{r}} \leq C(1-\lambda)^{\frac{1}{2}}\|f\|_{\left(X_{\lambda}, D\left(A_{\lambda}\right)\right)_{\sigma, 1}},
$$

where $C$ is independent of $\lambda$; see [11, Section 2.2] for details. So we have

$$
\begin{aligned}
\left\|\left(-\mathcal{L}_{\lambda}\right)^{-\frac{1}{2}} B(f, h)\right\|_{X_{\lambda}} & \leq C(1-\lambda)^{\frac{1}{4}\left(\frac{1}{2}-\frac{1}{q}\right)+\frac{3}{8}}\|f\|_{X_{\lambda}}^{\frac{1}{4}}\|f\|_{\left(X_{\lambda}, D\left(A_{\lambda}\right)\right)_{\sigma, 1}}^{\frac{3}{4}}\|h\|_{X_{\lambda}} \\
& \leq C(1-\lambda)^{\frac{1}{2}-\frac{3 \sigma}{8}}\|f\|_{D\left(A_{\lambda}^{\gamma}\right)}^{\frac{1}{4}}\|f\|_{\left(X_{\lambda}, D\left(A_{\lambda}\right)\right)_{\sigma, 1}}^{\frac{3}{4}}\|h\|_{X_{\lambda}}
\end{aligned}
$$

We also have

$$
\|f\|_{\left(X_{\lambda}, D\left(A_{\lambda}\right)\right)_{\sigma, 1}} \leq C\left(\frac{1}{\gamma-\sigma}+\frac{1}{\sigma}\right)\|f\|_{\left(X_{\lambda}, D\left(A_{\lambda}\right)\right)_{\gamma, \infty}}
$$

for $\gamma>\sigma$ by [11, Proposition 1.2.3], and

$$
\|f\|_{\left(X_{\lambda}, D\left(A_{\lambda}\right)\right)_{\gamma, \infty}} \leq C(\lambda)\|f\|_{D\left(A_{\lambda}^{\gamma}\right)}
$$

by [11, Proposition 2.2.15]. Thus we obtain

$$
\left\|\left(-\mathcal{L}_{\lambda}\right)^{-\frac{1}{2}} B(f, h)\right\|_{X_{\lambda}} \leq C(\lambda, \gamma)\|f\|_{D\left(A_{\lambda}^{\gamma}\right)}\|h\|_{X_{\lambda}} .
$$

This completes the proof.

\section{Existence of A Right SKEW-SyMmetrizer of $\Lambda$}

In this section we show the existence of a right skew-symmetrizer of $\Lambda$ in $\mathbb{P}^{e} X_{\lambda}=$ $\oplus_{n \in \mathbb{Z}} \mathbb{P}_{2 n} X_{\lambda}$. The representation of $\Lambda$ given in the previous section is essentially used. We start from the observation for the inverse of $\Lambda_{a}$.

From the definition of $\Lambda_{a, n}$ in (2.14), its inverse is given by

$$
\Lambda_{a, n}^{-1} f=-\frac{i}{n} \varphi^{-1} f, \quad|n| \geq 1 .
$$

We recall that Ker $\Lambda_{a}=\mathbb{P}_{0} X_{\lambda}$ by Proposition 2.2. Hence the inverse of $\Lambda_{a}$ in $\left(\operatorname{Ker} \Lambda_{a}\right)^{\perp}=\oplus_{|n| \geq 1} \mathbb{P}_{n} X_{\lambda}=\mathbb{P}_{0}^{\perp} X_{\lambda}$ is given by

$$
\Lambda_{a}^{-1} w=\sum_{|n| \geq 1} \Lambda_{a, n}^{-1}\left(w_{n}\right) e^{i n \theta}, \quad D\left(\Lambda_{a}^{-1}\right)=\left\{w \in \mathbb{P}_{0}^{\perp} X_{\lambda} \mid \sum_{|n| \geq 1} n^{-2}\left\|\varphi^{-1} w_{n}\right\|_{Z_{\lambda}}^{2}<\infty\right\} .
$$

From this clear representation of $\Lambda_{a}^{-1}$ we have the following important proposition.

Proposition 3.1. It follows that $\operatorname{Ran} \Lambda_{b} \subset D\left(\Lambda_{a}^{-1}\right)$. Moreover the operator $\Lambda_{a}^{-1} \Lambda_{b}$ satisfies that

$$
\left\|\Lambda_{a}^{-1} \Lambda_{b} w\right\|_{Y_{\lambda} \cap W_{\lambda}} \leq C\|w\|_{X_{\lambda}},
$$

where $C$ depends only on $\lambda$. Especially, $\Lambda_{a}^{-1} \Lambda_{b}$ is compact in $X_{\lambda}$. 
Proof. We note that $\Lambda_{b}$ maps $\mathbb{P}_{n} X_{\lambda}$ into $\mathbb{P}_{n} X_{\lambda}$ and $\Lambda_{b} \equiv 0$ on $\mathbb{P}_{0} X_{\lambda}$. Thus we see $\operatorname{Ran} \Lambda_{b} \subset \mathbb{P}_{0}^{\perp} X_{\lambda}$.

First let us prove that for any $f \in Z_{\lambda}$ and $r>0$,

$$
\begin{aligned}
&\left|\Omega_{n}(f)(r)\right| \leq \frac{C_{1, n}(r)}{|n|}\|f\|_{Z_{\lambda}}, \\
&\left|\Omega_{n}^{\prime}(f)(r)\right| \leq C_{2, n}(r)\|f\|_{Z_{\lambda}} .
\end{aligned}
$$

Here the positive functions $C_{1, n}(r)$ and $C_{2, n}(r)$ satisfy

$$
\begin{aligned}
\sup _{r>0}\left(r+(r|\log r|)^{-1}\right) C_{1,1}(r) & <\infty, \\
\sup _{r>0}\left(r^{2}+|\log r|^{-1}\right) C_{2,1}(r) & <\infty, \\
\sup _{r>0}\left(r+r^{-1}\right) C_{1, n}(r) & \leq C_{1},|n| \geq 2, \\
\sup _{r>0}\left(r^{2}+1\right) C_{2, n}(r) & \leq C_{2},|n| \geq 2,
\end{aligned}
$$

where $C_{1}$ and $C_{2}$ depend only on $\lambda$. Especially, $C_{1}$ and $C_{2}$ can be taken as independent of $n$.

We consider the estimate (3.4). From the representation (2.17), we see by the Hölder inequality,

$$
\begin{aligned}
\left|\Omega_{n}(f)(r)\right| \leq & \frac{1}{4|n|}\left\{r^{-|n|}\left(\int_{0}^{r} s^{2|n|+1} g_{\lambda}(s) d s\right)^{\frac{1}{2}}\left(\int_{0}^{\infty} r g_{\lambda}^{-1}(r)|f(r)|^{2} d r\right)^{\frac{1}{2}}\right. \\
& \left.+r^{|n|}\left(\int_{r}^{\infty} s^{1-2|n|} g_{\lambda}(s) d s\right)^{\frac{1}{2}}\left(\int_{0}^{\infty} r g_{\lambda}^{-1}(r)|f(r)|^{2} d r\right)^{\frac{1}{2}}\right\} \\
= & \frac{C_{1, n}(r)}{|n|}\|f\|_{Z_{\lambda},}
\end{aligned}
$$

where

$$
C_{1, n}(r):=\frac{1}{4}\left\{r^{-|n|}\left(\int_{0}^{r} s^{2|n|+1} g_{\lambda}(s) d s\right)^{\frac{1}{2}}+r^{|n|}\left(\int_{r}^{\infty} s^{1-2|n|} g_{\lambda}(s) d s\right)^{\frac{1}{2}}\right\} .
$$

It is not difficult to obtain the estimates (3.6) and (3.8) for the above $C_{1, n}(r)$. We omit the details here. As for the estimate (3.5), we observe that

$$
\Omega^{\prime}(f)(r)=-\frac{r^{-|n|-1}}{4} \int_{0}^{r} s^{|n|+1} f(s) d s+\frac{r^{|n|-1}}{4} \int_{r}^{\infty} s^{-|n|+1} f(s) d s .
$$

Hence as same as above, we have

$$
\left|\Omega^{\prime}(f)(r)\right| \leq\left. C_{2, n}(r)|| f\right|_{Z_{\lambda}}
$$

where

$$
C_{2, n}(r):=\frac{1}{4}\left\{r^{-|n|-1}\left(\int_{0}^{r} s^{2|n|+1} g_{\lambda}(s) d s\right)^{\frac{1}{2}}+r^{|n|-1}\left(\int_{r}^{\infty} s^{-2|n|+1} g_{\lambda}(s) d s\right)^{\frac{1}{2}}\right\},
$$

which gives the estimates (3.7) and (3.9). From the estimates (3.4) and (3.5), we can check that $\Lambda_{b} w \in D\left(\Lambda_{a}\right)$ for any $w \in X_{\lambda}$. 
Next we prove the estimate (3.3). We recall the relation $\Lambda_{a}^{-1} \Lambda_{b} \mathbb{P}_{n} w=\Lambda_{a, n}^{-1} \Lambda_{b, n}\left(w_{n}\right) e^{i n \theta}$ where $\mathbb{P}_{n} w=w_{n}(r) e^{i n \theta}$ as stated previously. From the definitions of $\Lambda_{a, n}$ and $\Lambda_{b, n}$, we see

$$
\Lambda_{a}^{-1} \Lambda_{b} \mathbb{P}_{n} w=-\frac{g}{\varphi} \Omega_{n}\left(w_{n}\right) e^{i n \theta}
$$

and

$$
\Lambda_{a}^{-1} \Lambda_{b} w=-\sum_{|n| \geq 1} \frac{g}{\varphi} \Omega_{n}\left(w_{n}\right) e^{i n \theta}
$$

We note that

$$
\begin{aligned}
\partial_{1} \Lambda_{a}^{-1} \Lambda_{b} \mathbb{P}_{n} w & =\left(\cos \theta \partial_{r}-\frac{\sin \theta}{r} \partial_{\theta}\right) \Lambda_{a}^{-1} \Lambda_{b} \mathbb{P}_{n} w \\
& =-\left(\frac{g^{\prime} \varphi-g \varphi^{\prime}}{\varphi^{2}} \Omega_{n}\left(w_{n}\right)+\frac{g}{\varphi} \Omega_{n}^{\prime}\left(w_{n}\right)\right) \cos \theta e^{i n \theta}+\frac{i n \sin \theta}{r} \frac{g}{\varphi} \Omega_{n}\left(w_{n}\right) e^{i n \theta} .
\end{aligned}
$$

Hence we have

$$
\left|\partial_{1} \Lambda_{a}^{-1} \Lambda_{b} \mathbb{P}_{n} w\right| \leq\left(\frac{\left|g^{\prime}\right|}{\varphi}+\frac{g\left|\varphi^{\prime}\right|}{\varphi^{2}}\right)\left|\Omega_{n}\left(w_{n}\right)\right|+\frac{g}{\varphi}\left|\Omega_{n}^{\prime}\left(w_{n}\right)\right|+\frac{|n|}{r} \frac{g}{\varphi}\left|\Omega_{n}\left(w_{n}\right)\right|
$$

Since $\varphi(r)=\frac{1}{2 \pi r^{2}}\left(1-e^{-\frac{r^{2}}{4}}\right)$, we see that $\varphi$ is positive and strictly decreasing with the order $r^{-2}$, and that $\varphi^{\prime}$ is bounded. So combining (3.13) with (3.4) and (3.5), we obtain

$$
\left\|\partial_{1} \Lambda_{a}^{-1} \Lambda_{b} \mathbb{P}_{n} w\right\|_{X_{\lambda}} \leq C|| w_{n} \|_{Z_{\lambda}}
$$

where $C$ is depending only on $\lambda$ and independent of $n$. We easily verify the relation $\left\|\partial_{1} \Lambda_{a}^{-1} \Lambda_{b} w\right\|_{X_{\lambda}}^{2} \leq C \sum_{|n| \geq 1}\left\|\partial_{1} \Lambda_{a}^{-1} \Lambda_{b} \mathbb{P}_{n} w\right\|_{X_{\lambda}}^{2}$. Thus we have $\left\|\partial_{1} \Lambda_{a}^{-1} \Lambda_{b} w\right\|_{X_{\lambda}}^{2} \leq$ $C\|w\|_{X_{\lambda}}^{2}$ where $C$ depends only on $\lambda$. Similarly we can obtain the estimates for $\partial_{2} \Lambda_{a}^{-1} \Lambda_{b} w$ and $|x| \Lambda_{a}^{-1} \Lambda_{b} w$. Now the proof of Proposition 3.1 is completed.

As a corollary of the above propositions, we have an important property of $I+$ $\Lambda_{a}^{-1} \Lambda_{b}$

Corollary 3.1. The operator $I+\Lambda_{a}^{-1} \Lambda_{b}$ is invertible in $\mathbb{P}^{e} X_{\lambda}$ and its inverse is a bounded operator on $\mathbb{P}^{e} X_{\lambda}$.

Proof. We note that $I+\Lambda_{a}^{-1} \Lambda_{b}$ maps $\mathbb{P}^{e} X_{\lambda}$ into $\mathbb{P}^{e} X_{\lambda}$. Since $\Lambda_{a}^{-1} \Lambda_{b}$ is a compact operator in $X_{\lambda}$, it suffices to prove that $I+\Lambda_{a}^{-1} \Lambda_{b}$ is injective in $\mathbb{P}^{e} X_{\lambda}$ by the Fredholm alternative theorem.

Let $\left(I+\Lambda_{a}^{-1} \Lambda_{b}\right) w=0$. Then from the relation $w=-\Lambda_{a}^{-1} \Lambda_{b} w$, we see that $w \in D\left(\Lambda_{a}\right)$. So we have $0=\Lambda_{a}\left(I+\Lambda_{a}^{-1} \Lambda_{b}\right) w=\Lambda_{a} w+\Lambda_{b} w=\Lambda w$.

By Proposition 2.2, the function $w$ belongs to $\mathbb{P}_{0} X_{\lambda} \oplus\left\{\beta_{1} \partial_{1} G+\beta_{2} \partial_{2} G \mid \beta_{i} \in \mathbb{R}\right\}$. However, since $w$ belongs to $\mathbb{P}^{e} X_{\lambda}$, we see that $w$ must belong to $\mathbb{P}_{0} X_{\lambda}$. On the other hand, we have $\mathbb{P}_{0} X_{\lambda} \subset \operatorname{Ker} \Lambda_{a}^{-1} \Lambda_{b}$ by the definition of $\Lambda_{b}$. Hence it follows that for any $w \in \operatorname{Ker}\left(I+\Lambda_{a}^{-1} \Lambda_{b}\right) \cap \mathbb{P}^{e} X_{\lambda}$, we have $w=\left(I+\Lambda_{a}^{-1} \Lambda_{b}\right) w-\Lambda_{a}^{-1} \Lambda_{b} w=0$. This completes the proof. 
Remark 3.1. If we calculate $C_{1, n}(r)$ in the proof of Proposition 3.1 precisely, we can show $\sup _{r>0} C_{1, n}(r) \leq \frac{\sqrt{2}}{4}$ for any $n \in \mathbb{Z}$ and $\lambda \in[0,1)$. Hence we have

$$
\begin{aligned}
\left\|\Lambda_{a}^{-1} \Lambda_{b} w\right\|_{X_{\lambda}}^{2}=\sum_{|n| \geq 1}\left\|\frac{g}{\varphi} \Omega_{n}\left(w_{n}\right) e^{i n \theta}\right\|_{X_{\lambda}}^{2} & \leq \sum_{|n| \geq 1}\left\|g \varphi^{-1} \Omega_{n}\left(w_{n}\right)\right\|_{Z_{\lambda}}^{2} \\
& \leq C\left\|g \varphi^{-1}\right\|_{Z_{\lambda}}^{2} \sum_{|n| \geq 1}\left\|w_{n}\right\|_{Z_{\lambda}}^{2} \\
& =C\left\|g \varphi^{-1}\right\|_{Z_{\lambda}}^{2}\|w\|_{X_{\lambda}}^{2},
\end{aligned}
$$

where $C$ is independent of $\lambda$. We note that $\left\|g \varphi^{-1}\right\|_{Z_{\lambda}}$ goes to infinity as $\lambda$ tends to 1 since $g_{\lambda}^{-1}=\frac{4 \pi}{1-\lambda} e^{\frac{1-\lambda}{4} r^{2}}$. This suggests that the operator norm $\left\|\Lambda_{a}^{-1} \Lambda_{b}\right\|_{X_{\lambda} \rightarrow X_{\lambda}}$ becomes larger as $\lambda$ tends to 1 . So we can not use perturbation arguments in order to prove the existence of the bounded inverse of $I+\Lambda_{a}^{-1} \Lambda_{b}$ (that is, the operator $\Lambda_{a}^{-1} \Lambda_{b}$ can not be regarded as the small perturbation of the operator I). Hence the use of the Fredholm alternative theorem based on the characterization of Ker $\Lambda$ is technically essential.

Proof of Lemma 1.1. The operator $\Lambda$ can be written as

$$
\Lambda=\Lambda_{a}\left(I+\Lambda_{a}^{-1} \Lambda_{b}\right)
$$

It is not difficult to see that $\Lambda_{a}$ is skew-symmetric, i.e.,

$$
<\Lambda_{a} f, h>_{X_{\lambda}}+<f, \Lambda_{a} h>_{X_{\lambda}}=0 \text {, for } f, h \in D\left(\Lambda_{a}\right) .
$$

In fact, we can also show that $\Lambda_{a}^{*}=-\Lambda_{a}$, but we omit the details here. From Corollary 3.1 it follows that $\Lambda\left(I+\Lambda_{a}^{-1} \Lambda_{b}\right)^{-1}=\Lambda_{a}$ in $\mathbb{P}^{e} X_{\lambda}$. Hence $\Lambda$ is right skewsymmetrizable in $\mathbb{P}^{e} X_{\lambda}$, and the operator $\left(I+\Lambda_{a}^{-1} \Lambda_{b}\right)^{-1}$ is a right skew-symmetrizer of $\Lambda$. It is clear that $T=\left(I+\Lambda_{a}^{-1} \Lambda_{b}\right)^{-1}$ satisfies the properties of Lemma 1.1 since $T-I=\Lambda_{a}^{-1} \Lambda_{b} T$ is compact. From the construction of $T$, we also see that $\operatorname{Ker} \Lambda T=\operatorname{Ker} \Lambda_{a}=\mathbb{P}_{0} X_{\lambda}$ and $T \mathbb{P}_{0} f=\mathbb{P}_{0} f$.

\section{THE LINEARIZED PROBLEM}

In the followoing sections the function spaces $X_{\lambda}, Y_{\lambda}$, and $W_{\lambda}$ are assumed to be real Hilbert spaces, and we always deal with real valued functions. In this section we consider the equation $\mathcal{L}_{\lambda, \alpha} h_{1}=f_{1}$ for a given $f_{1} \in \mathbb{P}^{e} X_{\lambda}$. Here $\mathcal{L}_{\lambda, \alpha}=\mathcal{L}+\lambda \mathcal{M}-\alpha \Lambda$. The main result is as follows.

Lemma 4.1. Let $\lambda \in[0,1)$ and $\gamma \in[0,1)$. Then there is a number $\Theta=\Theta(\lambda) \geq 0$ such that for any $\alpha \in \mathbb{R}$ with $|\alpha| \geq \Theta$, we have for $f_{1} \in \mathbb{P}^{e} X_{\lambda}$,

$$
\left\|\mathcal{L}_{\lambda, \alpha}^{-1} f_{1}\right\|_{Y_{\lambda} \cap W_{\lambda}} \leq J_{1}(\lambda)\left\|\left(-\mathcal{L}_{\lambda}\right)^{-\frac{1}{2}} f_{1}\right\|_{X \lambda}
$$

$$
\begin{aligned}
& \left\|\mathbb{P}_{0}^{\perp} \mathcal{L}_{\lambda, \alpha}^{-1} f_{1}\right\|_{D\left(A_{\lambda}^{\gamma}\right)} \leq \delta_{1}(|\alpha|, \lambda, \gamma)\left(\left\|\mathbb{P}_{0}^{\perp}\left(-\mathcal{L}_{\lambda}\right)^{-\frac{1}{2}} f_{1}\right\|_{X_{\lambda}}+\lambda\left\|\left(-\mathcal{L}_{\lambda}\right)^{-\frac{1}{2}} f_{1}\right\|_{X_{\lambda}}\right) \\
& \left\|\mathbb{P}_{0} \mathcal{L}_{\lambda, \alpha}^{-1} f_{1}\right\|_{D\left(A_{\lambda}^{\gamma}\right)} \leq J_{2}(\lambda)\left\|\mathbb{P}_{0}\left(-\mathcal{L}_{\lambda}\right)^{-\frac{1}{2}} f_{1}\right\|_{X_{\lambda}}+\lambda \delta_{2}(|\alpha|, \lambda, \gamma)\left\|\left(-\mathcal{L}_{\lambda}\right)^{-\frac{1}{2}} f_{1}\right\|_{X_{\lambda}}
\end{aligned}
$$


Here the constants $J_{1}$ and $J_{2}$ are independent of $\gamma$ and $\alpha$ with $|\alpha| \geq \Theta$. For each $\lambda \in[0,1)$ the constants $\delta_{1}(|\alpha|, \lambda, \gamma)$ and $\delta_{2}(|\alpha|, \lambda, \gamma)$ are bounded with respect to $|\alpha| \in[\Theta, \infty)$ and $\gamma \in[0,1)$, and satisfy that

$$
\lim _{|\alpha| \rightarrow \infty} \delta_{1}(|\alpha|, \lambda, \gamma)=\lim _{|\alpha| \rightarrow \infty} \delta_{2}(|\alpha|, \lambda, \gamma)=0
$$

The constants $\Theta, J_{1}$, and $J_{2}$ are taken as

$$
\lim _{\lambda \rightarrow 1} \Theta(\lambda)=\lim _{\lambda \rightarrow 1} J_{i}(\lambda)=\infty, i=1,2 .
$$

To prove this lemma we consider the equation for $h_{2}=T^{-1} h_{1}$ where $T=(I+$ $\left.\Lambda_{a}^{-1} \Lambda_{b}\right)^{-1}$ is the right skew-symmetrizer of $\Lambda$ in $\mathbb{P}^{e} X_{\lambda}$ constructed in Lemma 1.1. Then by the relation

$$
(\mathcal{L}+\lambda \mathcal{M}) h_{1}=(\mathcal{L}+\lambda \mathcal{M}) T h_{2}=(\mathcal{L}+\lambda \mathcal{M}) h_{2}+(\mathcal{L}+\lambda \mathcal{M})(T-I) h_{2}
$$

and

$$
\Lambda h_{1}=\Lambda T h_{2}=\Lambda_{a} h_{2},
$$

we obtain the equation for $h_{2}$ such as

$$
\left(\mathcal{L}+\lambda \mathcal{M}-\alpha \Lambda_{a}\right) h_{2}=(\mathcal{L}+\lambda \mathcal{M})(I-T) h_{2}+f_{1}
$$

Here $\Lambda_{a}$ is given by (2.18). Set

$$
L_{\lambda, \alpha}=\mathcal{L}+\lambda \mathcal{M}-\alpha \Lambda_{a}
$$

and we first consider the linear problem

$$
L_{\lambda, \alpha} h=f
$$

for a given $f \in \mathbb{P}^{e} X_{\lambda}$.

We recall that $\mathcal{L}=\mathcal{L}_{\lambda}+\lambda \mathcal{N}$ and the spectrum of the self-adjoint operator $\mathcal{L}_{\lambda}$ in $X_{\lambda}$ is given by $\left\{-\frac{(1-\lambda) n}{2} \mid n=1,2, \cdots\right\}$. We first consider the operator $L_{\lambda, \alpha}-\lambda I$ and establish some estimates for $\left(L_{\lambda, \alpha}-\lambda I\right)^{-1}$. Then we consider the equation $\left(L_{\lambda, \alpha}-\lambda I\right) h=-\lambda h+f$. The key steps here are based on contradiction arguments that are already used in [14]. But we need further idea to obtain desired estimates for solutions of $\left(L_{\lambda, \alpha}-\lambda I\right) h=-\lambda h+f$ when $\lambda$ is near 1 , because the spectrum of $\mathcal{L}_{\lambda}$ approaches to 0 as $\lambda$ tends to 1 . We overcome this difficulty by using a cut-off technique and combining the estimates for the $X_{0}$-norm of solutions in a bounded domain and the estimates for the $X_{\lambda}$-norm of solutions in an unbounded domain. Since Ker $\Lambda_{a}=\mathbb{P}_{0} X_{\lambda}$, we decompose solutions into radially symmetric parts and non-radially symmetric parts, and prove that non-radially symmetric parts of solutions to $L_{\lambda, \alpha} h=f$ are estimated as small if $|\alpha|$ is sufficiently large. This is the most essential estimate in this section. More precisely, we shall prove the following lemma. 
Lemma 4.2. Let $\lambda \in[0,1)$ and $\gamma \in[0,1)$. Then there is a number $\Theta^{\prime}=\Theta^{\prime}(\lambda) \geq 0$ such that for any $\alpha \in \mathbb{R}$ with $|\alpha| \geq \Theta^{\prime}$, we have for $f \in \mathbb{P}^{e} X_{\lambda}$,

$$
\left\|L_{\lambda, \alpha}^{-1} f\right\|_{Y_{\lambda} \cap W_{\lambda}} \leq J_{1}(\lambda)\left\|\left(-\mathcal{L}_{\lambda}\right)^{-\frac{1}{2}} f\right\|_{X \lambda}
$$

$$
\left\|\mathbb{P}_{0}^{\perp} L_{\lambda, \alpha}^{-1} f\right\|_{D\left(A_{\lambda}^{\gamma}\right)} \leq \delta_{1}(|\alpha|, \lambda, \gamma)\left(\left\|\mathbb{P}_{0}^{\perp}\left(-\mathcal{L}_{\lambda}\right)^{-\frac{1}{2}} f\right\|_{X_{\lambda}}+\lambda\left\|\left(-\mathcal{L}_{\lambda}\right)^{-\frac{1}{2}} f\right\|_{X_{\lambda}}\right),
$$

$$
\left\|\mathbb{P}_{0} L_{\lambda, \alpha}^{-1} f\right\|_{D\left(A_{\lambda}^{\gamma}\right)} \leq J_{2}(\lambda)\left\|\mathbb{P}_{0}\left(-\mathcal{L}_{\lambda}\right)^{-\frac{1}{2}} f\right\|_{X_{\lambda}}+\lambda \delta_{2}(|\alpha|, \lambda, \gamma)\left\|\left(-\mathcal{L}_{\lambda}\right)^{-\frac{1}{2}} f\right\|_{X_{\lambda}} .
$$

Here the constants $J_{1}$ and $J_{2}$ are independent of $\gamma$ and $\alpha$ with $|\alpha| \geq \Theta^{\prime}$. For each $\lambda \in[0,1)$ the constants $\delta_{1}(|\alpha|, \lambda, \gamma)$ and $\delta_{2}(|\alpha|, \lambda, \gamma)$ are bounded with respect to $|\alpha| \in\left[\Theta^{\prime}, \infty\right)$ and $\gamma \in[0,1)$, and satisfy that

$$
\lim _{|\alpha| \rightarrow \infty} \delta_{1}(|\alpha|, \lambda, \gamma)=\lim _{|\alpha| \rightarrow \infty} \delta_{2}(|\alpha|, \lambda, \gamma)=0
$$

The constants $\Theta^{\prime}, J_{1}$, and $J_{2}$ are taken as

$$
\lim _{\lambda \rightarrow 1} \Theta^{\prime}(\lambda)=\lim _{\lambda \rightarrow 1} J_{i}(\lambda)=\infty, i=1,2 .
$$

Lemma 4.1 is obtained by applying Lemma 4.2 to (4.6).

The proof of Lemma 4.2 is decomposed into several steps below.

4.1. Estimates for $\left(L_{\lambda, \alpha}-\lambda I\right)^{-1}$. As stated above, we first consider the equation $\left(L_{\lambda, \alpha}-\lambda I\right) h=f$ for $f \in \mathbb{P}^{e} X_{\lambda}$. Let us start from the following proposition.

Proposition 4.1. Let $\lambda \in[0,1)$ and $\alpha \in \mathbb{R}$. Then the operator $L_{\lambda, \alpha}-\lambda I$ has a bounded inverse in $\mathbb{P}^{e} X_{\lambda}$ and we have for any $f \in \mathbb{P}^{e} X_{\lambda}$

$$
\left\|\left(L_{\lambda, \alpha}-\lambda I\right)^{-1} f\right\|_{Y_{\lambda} \cap W_{\lambda}} \leq \frac{C}{1-\lambda}\left\|\left(-\mathcal{L}_{\lambda}\right)^{-\frac{1}{2}} f\right\|_{X_{\lambda}} .
$$

Here the constant $C$ is independent of $\lambda$ and $\alpha$.

Proof of Proposition 4.1. The operator $L_{\lambda, \alpha}-\lambda I$ can be written as

$$
L_{\lambda, \alpha}-\lambda I=-\left(-\mathcal{L}_{\lambda}\right)^{\frac{1}{2}}\left(I-\lambda \Pi_{1}-\lambda \Pi_{2}+\alpha \Sigma_{a}+\lambda\left(-\mathcal{L}_{\lambda}\right)^{-1}\right)\left(-\mathcal{L}_{\lambda}\right)^{\frac{1}{2}}
$$

where

$$
\begin{aligned}
& \Pi_{1}=\left(-\mathcal{L}_{\lambda}\right)^{-\frac{1}{2}} \mathcal{M}\left(-\mathcal{L}_{\lambda}\right)^{-\frac{1}{2}}, \\
& \Pi_{2}=\left(-\mathcal{L}_{\lambda}\right)^{-\frac{1}{2}} \mathcal{N}\left(-\mathcal{L}_{\lambda}\right)^{-\frac{1}{2}}, \\
& \Sigma_{a}=\left(-\mathcal{L}_{\lambda}\right)^{-\frac{1}{2}} \Lambda_{a}\left(-\mathcal{L}_{\lambda}\right)^{-\frac{1}{2}} .
\end{aligned}
$$

It is not difficult to see that the operators $\Pi_{1}, \Pi_{2}$, and $\Sigma_{a}$ are bounded in $\mathbb{P}^{e} X_{\lambda}$. Indeed, from the definitions $\mathcal{M} f=\frac{1}{2}\left(\partial_{1} x_{1}-\partial_{2} x_{2}\right) f, \mathcal{N} f=\nabla \cdot\left(\frac{x}{2} f\right)$, and $\Lambda_{a} f=(K *$ $G, \nabla) f$ for $f \in Y_{\lambda} \cap W_{\lambda}$, each operator above is bounded in $\mathbb{P}^{e} X_{\lambda}$ from Proposition 2.1 and Proposition 2.4. We also note that since $\Lambda_{a}$ is skew-symmetric, so is $\Sigma_{a}$.

We define the bilinear form $Q_{\lambda, \alpha}$ in $\mathbb{P}^{e} X_{\lambda} \times \mathbb{P}^{e} X_{\lambda}$ as

$$
Q_{\lambda, \alpha}(f, h)=<\left(I-\lambda \Pi_{1}-\lambda \Pi_{2}+\alpha \Sigma_{a}+\lambda\left(-\mathcal{L}_{\lambda}\right)^{-1}\right) f, h>_{X_{\lambda}} .
$$


Then it is easy to see that $Q_{\lambda, \alpha}$ is bounded, i.e., there is a positive $C(\lambda, \alpha)$ such that $\left|Q_{\lambda, \alpha}(f, h)\right| \leq C(\lambda, \alpha)\|f\|_{X_{\lambda}}\|h\|_{X_{\lambda}}$ holds for any $f, h \in X_{\lambda}$.

Moreover we have by skew-symmetry of $\Sigma_{a}$,

$$
\begin{aligned}
& Q_{\lambda, \alpha}(f, f) \\
= & \|f\|_{X_{\lambda}}^{2}-\lambda<\Pi_{1} f, f>_{X_{\lambda}}-\lambda<\Pi_{2} f, f>_{X_{\lambda}}+\lambda<\left(-\mathcal{L}_{\lambda}\right)^{-1} f, f>_{X_{\lambda}} \\
= & \|f\|_{X_{\lambda}}^{2}-\lambda<\mathcal{M}\left(-\mathcal{L}_{\lambda}\right)^{-\frac{1}{2}} f,\left(-\mathcal{L}_{\lambda}\right)^{-\frac{1}{2}} f>_{X_{\lambda}}-\lambda<\frac{x}{2} \cdot \nabla\left(-\mathcal{L}_{\lambda}\right)^{-\frac{1}{2}} f,\left(-\mathcal{L}_{\lambda}\right)^{-\frac{1}{2}} f>_{X_{\lambda}} .
\end{aligned}
$$

Set $h=\left(-\mathcal{L}_{\lambda}\right)^{-\frac{1}{2}} f$. Then we see by the integration by parts,

$$
\begin{aligned}
& <\mathcal{M}\left(-\mathcal{L}_{\lambda}\right)^{-\frac{1}{2}} f,\left(-\mathcal{L}_{\lambda}\right)^{-\frac{1}{2}} f>_{X_{\lambda}}+<\frac{x}{2} \cdot \nabla\left(-\mathcal{L}_{\lambda}\right)^{-\frac{1}{2}} f,\left(-\mathcal{L}_{\lambda}\right)^{-\frac{1}{2}} f>_{X_{\lambda}} \\
= & \int_{\mathbb{R}^{2}} G_{\lambda}^{-1} h \frac{1}{2}\left(x_{1} \partial_{1}-x_{2} \partial_{2}\right) h d x+\int_{\mathbb{R}^{2}} G_{\lambda}^{-1} h \frac{x}{2} \cdot \nabla h d x \\
= & -\frac{1}{2} \int_{\mathbb{R}^{2}} G_{\lambda}^{-1}|h|^{2} d x-\frac{1-\lambda}{2} \int_{\mathbb{R}^{2}} G_{\lambda}^{-1} x_{1}^{2}|h|^{2} d x .
\end{aligned}
$$

Hence we have

$$
\begin{aligned}
Q_{\lambda, \alpha}(f, f) & =\|f\|_{X_{\lambda}}^{2}+\frac{\lambda}{2}\left\|\left(-\mathcal{L}_{\lambda}\right)^{-\frac{1}{2}} f\right\|_{X_{\lambda}}^{2}+\frac{\lambda(1-\lambda)}{2}\left\|x_{1}\left(-\mathcal{L}_{\lambda}\right)^{-\frac{1}{2}} f\right\|_{X_{\lambda}}^{2} \\
& \geq\|f\|_{X_{\lambda}}^{2} .
\end{aligned}
$$

Thus by the Lax-Milgram theorem, the operator $I-\lambda \Pi_{1}-\lambda \Pi_{2}+\alpha \Sigma_{a}+\lambda\left(-\mathcal{L}_{\lambda}\right)^{-1}$ is invertible and

$$
\left\|\left(I-\lambda \Pi_{1}-\lambda \Pi_{2}+\alpha \Sigma_{a}+\lambda\left(-\mathcal{L}_{\lambda}\right)^{-1}\right)^{-1} f\right\|_{X_{\lambda}} \leq \mid f \|_{X_{\lambda}} .
$$

Combining this with Proposition 2.1, we have the desired estimate (4.14). The proof of Proposition 4.1 is now completed.

Let $h=\left(L_{\lambda, \alpha}-\lambda I\right)^{-1} \tilde{f}$ for $\tilde{f} \in \mathbb{P}^{e} X_{\lambda}$. Then we have $\left(\mathcal{L}_{\lambda}+\lambda \mathcal{N}-\alpha \Lambda_{a}-\lambda I\right) h=$ $\tilde{f}-\lambda \mathcal{M} h$. Thus $h$ is written as

$$
h=-\left(-\mathcal{L}_{\lambda}\right)^{-\frac{1}{2}} \Gamma_{\lambda, \alpha}^{-1}\left(-\mathcal{L}_{\lambda}\right)^{-\frac{1}{2}} \tilde{f}+\lambda\left(-\mathcal{L}_{\lambda}\right)^{-\frac{1}{2}} \Gamma_{\lambda, \alpha}^{-1}\left(-\mathcal{L}_{\lambda}\right)^{-\frac{1}{2}} \mathcal{M} h,
$$

where

$$
\Gamma_{\lambda, \alpha}=I-\lambda \Pi_{2}+\alpha \Sigma_{a}+\lambda\left(-\mathcal{L}_{\lambda}\right)^{-1} .
$$

Here we used the relation

$$
\left(\mathcal{L}_{\lambda}+\lambda \mathcal{N}-\alpha \Lambda_{a}-\lambda I\right)^{-1}=-\left(-\mathcal{L}_{\lambda}\right)^{-\frac{1}{2}} \Gamma_{\lambda, \alpha}^{-1}\left(-\mathcal{L}_{\lambda}\right)^{-\frac{1}{2}}
$$

Next we decompose $h$ into the radially symmetric part and the non-radially symmetric part. Note that the projection $\mathbb{P}_{0}$ or $\mathbb{P}_{0}^{\perp}$ commutes with the operators $\left(-\mathcal{L}_{\lambda}\right)^{-\frac{1}{2}}$ and $\Gamma_{\lambda, \alpha}^{-1}$, and that $\mathbb{P}_{0} \Gamma_{\lambda, \alpha}=\Gamma_{\lambda, \alpha} \mathbb{P}_{0}=\Gamma_{\lambda, 0} \mathbb{P}_{0}$ since Ker $\Sigma_{a}=\mathbb{P}_{0} X_{\lambda}$. The same is true for $\Gamma_{\lambda, \alpha}^{-1}$. For simplicity we write $\mathbb{P}_{0} h=h_{S}$ and $\mathbb{P}_{0}^{\perp} h=h_{S}^{\perp}$. Then we have from (4.20), 


$$
\begin{aligned}
& h_{S}=-\left(-\mathcal{L}_{\lambda}\right)^{-\frac{1}{2}} \Gamma_{\lambda, 0}^{-1} \mathbb{P}_{0}\left(-\mathcal{L}_{\lambda}\right)^{-\frac{1}{2}} \tilde{f}+\lambda\left(-\mathcal{L}_{\lambda}\right)^{-\frac{1}{2}} \Gamma_{\lambda, 0}^{-1} \mathbb{P}_{0}\left(-\mathcal{L}_{\lambda}\right)^{-\frac{1}{2}} \mathcal{M} h_{S}^{\perp}, \\
& h_{S}^{\perp}=-\left(-\mathcal{L}_{\lambda}\right)^{-\frac{1}{2}} \Gamma_{\lambda, \alpha}^{-1} \mathbb{P}_{0}^{\perp}\left(-\mathcal{L}_{\lambda}\right)^{-\frac{1}{2}} \tilde{f}+\lambda\left(-\mathcal{L}_{\lambda}\right)^{-\frac{1}{2}} \Gamma_{\lambda, \alpha}^{-1} \mathbb{P}_{0}^{\perp}\left(-\mathcal{L}_{\lambda}\right)^{-\frac{1}{2}} \mathcal{M} h .
\end{aligned}
$$

Here we used the fact that $\mathbb{P}_{0}\left(-\mathcal{L}_{\lambda}\right)^{-\frac{1}{2}} \mathcal{M} h=\mathbb{P}_{0}\left(-\mathcal{L}_{\lambda}\right)^{-\frac{1}{2}} \mathcal{M} \mathbb{P}_{0}^{\perp} h$ by the property of the operator $\mathcal{M}=\frac{1}{2}\left(x_{1} \partial_{1}-x_{2} \partial_{2}\right)$.

We recall that $\Pi_{1}=\left(-\mathcal{L}_{\lambda}\right)^{-\frac{1}{2}} \mathcal{M}\left(-\mathcal{L}_{\lambda}\right)^{-\frac{1}{2}}$, and that $A_{\lambda}=\left(-\mathcal{L}_{\lambda}\right)^{\frac{1}{2}}$ and $D\left(A_{\lambda}^{\gamma}\right)$ is equipped with the norm $\|f\|_{D\left(A_{\lambda}^{\gamma}\right)}=\|f\|_{X_{\lambda}}+\left\|\left(-\mathcal{L}_{\lambda}\right)^{\frac{\gamma}{2}} f\right\|_{X_{\lambda}}$ which is equivalent with $\left\|\left(-\mathcal{L}_{\lambda}\right)^{\frac{\gamma}{2}} f\right\|_{X_{\lambda}}$ itself. Then we have the following important proposition.

Proposition 4.2. Let $\lambda \in[0,1)$ and $\gamma \in[0,1)$. Then we have for any $f \in \mathbb{P}^{e} X_{\lambda}$,

$$
\begin{aligned}
& \left\|\left(-\mathcal{L}_{\lambda}\right)^{-\frac{1}{2}} \Gamma_{\lambda, 0}^{-1} \mathbb{P}_{0} f\right\|_{D\left(A_{\lambda}^{\gamma}\right)} \leq(1-\lambda)^{\frac{\gamma-1}{2}}\left\|\mathbb{P}_{0} f\right\|_{X_{\lambda}}, \\
& \left\|\left(-\mathcal{L}_{\lambda}\right)^{-\frac{1}{2}} \Gamma_{\lambda, \alpha}^{-1} \mathbb{P}_{0}^{\perp} f\right\|_{D\left(A_{\lambda}^{\gamma}\right)} \leq \epsilon_{1}(|\alpha|, \lambda, \gamma)\left\|\mathbb{P}_{0}^{\perp} f\right\|_{X_{\lambda}}, \\
& \left\|\left(-\mathcal{L}_{\lambda}\right)^{-\frac{1}{2}} \Gamma_{\lambda, 0}^{-1} \mathbb{P}_{0} \Pi_{1} \Gamma_{\lambda, \alpha}^{-1} \mathbb{P}_{0}^{\perp} f\right\|_{D\left(A_{\lambda}^{\gamma}\right)} \leq \epsilon_{2}(|\alpha|, \lambda, \gamma)\left\|\mathbb{P}_{0}^{\perp} f\right\|_{X_{\lambda}} .
\end{aligned}
$$

For each $\lambda \in[0,1)$, the constants $\epsilon_{1}(|\alpha|, \lambda, \gamma)$ and $\epsilon_{2}(|\alpha|, \lambda, \gamma)$ are bounded with respect to $|\alpha|$ and $\gamma \in[0,1)$. Moreover we have

$$
\lim _{|\alpha| \rightarrow \infty} \epsilon_{i}(|\alpha|, \lambda, \gamma)=0, i=1,2 .
$$

Proof of Proposition 4.2. To prove the estimate (4.24), we first note that

$$
\left\|\left(-\mathcal{L}_{\lambda}\right)^{\frac{\gamma-1}{2}}\right\|_{X_{\lambda} \rightarrow X_{\lambda}} \leq(1-\lambda)^{\frac{\gamma-1}{2}}
$$

which is obtained by the estimate (2.2) and the interpolation inequality

$$
\left\|\left(-\mathcal{L}_{\lambda}\right)^{\frac{\gamma-1}{2}} f\right\|_{X_{\lambda}} \leq\left\|\left(-\mathcal{L}_{\lambda}\right)^{-\frac{1}{2}} f\right\|_{X_{\lambda}}^{1-\gamma}\|f\|_{X_{\lambda}}^{\gamma} .
$$

It is not difficult to see that

$$
\left\|\Gamma_{\lambda, \alpha}^{-1} f\right\|_{X_{\lambda}} \leq\|f\|_{X_{\lambda}}
$$

for any $\alpha \in \mathbb{R}$. Indeed, the bilinear form $\tilde{Q}_{\lambda, \alpha}(f, h)=<\Gamma_{\lambda, \alpha} f, h>_{X_{\lambda}}$ is bounded and satisfies that

$$
\begin{aligned}
\tilde{Q}_{\lambda, \alpha}(f, f) & =\|f\|_{X_{\lambda}}^{2}-\lambda<\Pi_{2} f, f>_{X_{\lambda}}+\lambda<\left(-\mathcal{L}_{\lambda}\right)^{-1} f, f>_{X_{\lambda}} \\
& =\|f\|_{X_{\lambda}}^{2}-\lambda<\frac{x}{2} \cdot \nabla\left(-\mathcal{L}_{\lambda}\right)^{-\frac{1}{2}} f,\left(-\mathcal{L}_{\lambda}\right)^{-\frac{1}{2}} f>_{X_{\lambda}} \\
& =\|f\|_{X_{\lambda}}^{2}+\lambda\left\|\left(-\mathcal{L}_{\lambda}\right)^{-\frac{1}{2}} f\right\|_{X_{\lambda}}^{2}+\frac{\lambda(1-\lambda)}{2}\left\|x \mid\left(-\mathcal{L}_{\lambda}\right)^{-\frac{1}{2}} f\right\|_{X_{\lambda}}^{2} \\
& \geq\|f\|_{X_{\lambda}}^{2} .
\end{aligned}
$$


By the Lax-Milgram theorem, we have the estimate (4.29). Thus the estimate (4.24) holds.

We prove the estimates (4.25) and (4.26) by deriving a contradiction. This argument is also used in [14, Lemma 3.2].

Without loss of generality, we may assume that $\alpha>0$. Set

$$
\epsilon_{1}(\alpha, \gamma)=\left\|\left(-\mathcal{L}_{\lambda}\right)^{-\frac{1}{2}} \Gamma_{\lambda, \alpha}^{-1} \mathbb{P}_{0}^{\perp}\right\|_{\mathbb{P}^{e} X_{\lambda} \rightarrow D\left(A_{\lambda}^{\gamma}\right)} .
$$

Then $\epsilon_{1}(\alpha, \gamma)$ is uniformly bounded with respect to $\alpha$ and $\gamma$ by the estimate (4.24).

We assume that $\lim \sup _{\alpha \rightarrow \infty} \epsilon_{1}(\alpha, \gamma)>0$. Then there exists a sequence $\left\{\alpha_{i}\right\}_{i \in \mathbb{N}}$, $\alpha_{i} \rightarrow \infty$ as $i \rightarrow \infty$, such that $\epsilon_{1}=\inf _{i \in \mathbb{N}} \epsilon_{1}\left(\alpha_{i}\right)>0$. So we have a sequence of functions $\left\{f_{i}\right\}_{i \in \mathbb{N}}$ with $\left\|f_{i}\right\|_{X_{\lambda}}=1$ such that

$$
\left\|\left(-\mathcal{L}_{\lambda}\right)^{-\frac{1}{2}} \Gamma_{\lambda, \alpha_{i}}^{-1} \mathbb{P}_{0}^{\perp} f_{i}\right\|_{D\left(A_{\lambda}^{\gamma}\right)} \geq \frac{\epsilon_{1}\left(\alpha_{i}\right)}{2}\left\|f_{i}\right\|_{X_{\lambda}} \geq \frac{\epsilon_{1}}{2}>0 .
$$

We set $h_{i}=\left(-\mathcal{L}_{\lambda}\right)^{\frac{-1+\gamma}{2}} \Gamma_{\lambda, \alpha_{i}}^{-1} \mathbb{P}_{0}^{\perp} f_{i} \in \mathbb{P}_{0}^{\perp} X_{\lambda}$.

Since $\left(-\mathcal{L}_{\lambda}\right)^{\frac{-1+\gamma}{2}}$ is compact (because $\left(-\mathcal{L}_{\lambda}\right)^{-\frac{1}{2}}$ is compact) and $\left\{\Gamma_{\lambda, \alpha_{i}}^{-1} \mathbb{P}_{0}^{\perp} f_{i}\right\}$ is bounded in $X_{\lambda}$, we have a subsequence $\left\{h_{j}\right\}$ of $\left\{h_{i}\right\}$ such that $h_{j}$ converges to a function $h_{\infty} \in \mathbb{P}_{0}^{\perp} X_{\lambda}$ strongly in $X_{\lambda}$. Then $h_{\infty}$ satisfies $\left(-\mathcal{L}_{\lambda}\right)^{\frac{1-\gamma}{2}} h_{\infty} \in \mathbb{P}_{0}^{\perp} X_{\lambda}$ and $\left\|h_{\infty}\right\|_{X_{\lambda}} \geq \frac{\epsilon_{1}}{2}>0$.

On the other hand, for any $f \in X_{\lambda}$, we see

$$
\begin{aligned}
& <\left(-\mathcal{L}_{\lambda}\right)^{-\frac{1}{2}} \Lambda_{a}\left(-\mathcal{L}_{\lambda}\right)^{-\frac{\gamma}{2}} h_{\infty}, f>_{X_{\lambda}} \\
= & -<\left(-\mathcal{L}_{\lambda}\right)^{-\frac{\gamma}{2}} h_{\infty}, \Lambda_{a}\left(-\mathcal{L}_{\lambda}\right)^{-\frac{1}{2}} f>_{X_{\lambda}} \\
= & -\lim _{j \rightarrow \infty}<\left(-\mathcal{L}_{\lambda}\right)^{-\frac{\gamma}{2}} h_{j}, \Lambda_{a}\left(-\mathcal{L}_{\lambda}\right)^{-\frac{1}{2}} f>_{X_{\lambda}} \\
= & \lim _{j \rightarrow \infty}<\left(-\mathcal{L}_{\lambda}\right)^{-\frac{1}{2}} \Lambda_{a}\left(-\mathcal{L}_{\lambda}\right)^{-\frac{\gamma}{2}} h_{j}, f>_{X_{\lambda}} \\
= & \lim _{j \rightarrow \infty} \frac{1}{\alpha_{j}}\left(<\Gamma_{\lambda, \alpha_{j}}\left(-\mathcal{L}_{\lambda}\right)^{\frac{1-\gamma}{2}} h_{j}, f>_{X_{\lambda}}-<\left(-\mathcal{L}_{\lambda}\right)^{\frac{1-\gamma}{2}} h_{j}, f>_{X_{\lambda}}\right. \\
& \left.+\lambda<\Pi_{2}\left(-\mathcal{L}_{\lambda}\right)^{\frac{1-\gamma}{2}} h_{j}, f>_{X_{\lambda}}-\lambda<\left(-\mathcal{L}_{\lambda}\right)^{-\frac{1+\gamma}{2}} h_{j}, f>_{X_{\lambda}}\right) \\
= & \lim _{j \rightarrow \infty} \frac{1}{\alpha_{j}}\left(<\mathbb{P}_{0}^{\perp} f_{j}, f>_{X_{\lambda}}-<\left(-\mathcal{L}_{\lambda}\right)^{\frac{1-\gamma}{2}} h_{j}, f>_{X_{\lambda}}\right. \\
& \left.+\lambda<\Pi_{2}\left(-\mathcal{L}_{\lambda}\right)^{\frac{1-\gamma}{2}} h_{j}, f>_{X_{\lambda}}-\lambda<\left(-\mathcal{L}_{\lambda}\right)^{-\frac{1+\gamma}{2}} h_{j}, f>_{X_{\lambda}}\right) \\
= & 0 .
\end{aligned}
$$

Thus $\left(-\mathcal{L}_{\lambda}\right)^{-\frac{1}{2}} \Lambda_{a}\left(-\mathcal{L}_{\lambda}\right)^{-\frac{\gamma}{2}} h_{\infty}=0$, that is,$\Lambda_{a}\left(-\mathcal{L}_{\lambda}\right)^{-\frac{\gamma}{2}} h_{\infty}=0$. However, since Ker $\Lambda_{a}=\mathbb{P}_{0} X_{\lambda}$ and $h_{\infty} \in \mathbb{P}_{0}^{\perp} X_{\lambda}$ (and thus $\left(-\mathcal{L}_{\lambda}\right)^{-\frac{\gamma}{2}} h_{\infty} \in \mathbb{P}_{0}^{\perp} X_{\lambda}$ ), we must have $\left(-\mathcal{L}_{\lambda}\right)^{-\frac{\gamma}{2}} h_{\infty}=0$. Hence $h_{\infty}=0$. This contradicts with $\left\|h_{\infty}\right\|_{X_{\lambda}}>0$. Now the estimate (4.25) has been proved.

The estimate (4.25) leads to the following simple claim.

Claim. Let $\left\{f_{i}\right\}_{i \in \mathbb{N}}$ be any bounded sequence in $\mathbb{P}^{e} X_{\lambda}$. Then for any sequence $\left\{\alpha_{i}\right\}_{i \in \mathbb{N}}$ in $\mathbb{R}$ such that $\alpha_{i} \rightarrow \infty$ as $i \rightarrow \infty$, the sequence $z_{i}=\Gamma_{\lambda, \alpha_{i}}^{-1} \mathbb{P}_{0}^{\perp} f_{i}$ weakly converges to 0 in $X_{\lambda}$. 
Indeed, for any $f \in D\left(\mathcal{L}_{\lambda}\right)$ we have

$$
\begin{aligned}
\lim _{i \rightarrow \infty}<z_{i}, f>_{X_{\lambda}} & =\lim _{i \rightarrow \infty}<z_{i},\left(-\mathcal{L}_{\lambda}\right)^{\frac{-1+\gamma}{2}}\left(-\mathcal{L}_{\lambda}\right)^{\frac{1-\gamma}{2}} f>_{X_{\lambda}} \\
& =\lim _{i \rightarrow \infty}<\left(-\mathcal{L}_{\lambda}\right)^{\frac{-1+\gamma}{2}} z_{i},\left(-\mathcal{L}_{\lambda}\right)^{\frac{1-\gamma}{2}} f>_{X_{\lambda}}=0
\end{aligned}
$$

Since $D\left(\mathcal{L}_{\lambda}\right)$ is dense in $X_{\lambda}$ and $\left\{z_{i}\right\}$ is bounded in $X_{\lambda}$ by the estimate (4.29), we have the claim.

The estimate (4.26) follows by the above claim. Indeed, we set

$$
\epsilon_{2}(\alpha, \gamma)=\left\|\left(-\mathcal{L}_{\lambda}\right)^{-\frac{1}{2}} \Gamma_{\lambda, 0}^{-1} \mathbb{P}_{0} \Pi_{1} \Gamma_{\lambda, \alpha}^{-1} \mathbb{P}_{0}^{\perp}\right\|_{\mathbb{P} e} X_{\lambda} \rightarrow D\left(A_{\lambda}^{\gamma}\right)
$$

Again we assume that there exists a sequence $\left\{\alpha_{i}\right\}_{i \in \mathbb{N}}, \alpha_{i} \rightarrow \infty$ as $i \rightarrow \infty$, satisfying $\epsilon_{2}=\inf _{i \in \mathbb{N}} \epsilon_{2}\left(\alpha_{i}, \gamma\right)>0$. Then we have $\left\{f_{i}\right\}_{i \in \mathbb{N}}$ with $\left\|f_{i}\right\|_{X_{\lambda}}=1$ such that the function $h_{i}$ given by

$$
h_{i}=\left(-\mathcal{L}_{\lambda}\right)^{\frac{-1+\gamma}{2}} \Gamma_{\lambda, 0}^{-1} \mathbb{P}_{0} \Pi_{1} \Gamma_{\lambda, \alpha_{i}}^{-1} \mathbb{P}_{0}^{\perp} f_{i}
$$

satisfies

$$
\begin{aligned}
\left\|h_{i}\right\|_{X_{\lambda}} & =\left\|\left(-\mathcal{L}_{\lambda}\right)^{\frac{\gamma}{2}}\left(-\mathcal{L}_{\lambda}\right)^{-\frac{\gamma}{2}} h_{i}\right\|_{X_{\lambda}} \\
& \geq C(\lambda)\left\|\left(-\mathcal{L}_{\lambda}\right)^{-\frac{\gamma}{2}} h_{i}\right\|_{D\left(A_{\lambda}^{\gamma}\right)} \\
& =C(\lambda)\left\|\left(-\mathcal{L}_{\lambda}\right)^{-\frac{1}{2}} \Gamma_{\lambda, 0}^{-1} \mathbb{P}_{0} \Pi_{1} \Gamma_{\lambda, \alpha}^{-1} \mathbb{P}_{0}^{\perp} f_{i}\right\|_{D\left(A_{\lambda}^{\gamma}\right)} \\
& \geq C(\lambda) \frac{\epsilon_{2}}{2}
\end{aligned}
$$

uniformly in $i \in \mathbb{N}$. Here we used the fact that $\|f\|_{D\left(A_{\lambda}^{\gamma}\right)}$ and $\left\|\left(-\mathcal{L}_{\lambda}\right)^{\frac{\gamma}{2}} f\right\|_{X_{\lambda}}$ are equivalent.

Since $\Pi_{1} \Gamma_{\lambda, \alpha_{i}}^{-1} \mathbb{P}_{0}^{\perp}$ is bounded and $\left(-\mathcal{L}_{\lambda}\right)^{\frac{-1+\gamma}{2}}$ is compact in $X_{\lambda}$, we have a subsequence $\left\{h_{j}\right\}_{j \in \mathbb{N}}$ of $\left\{h_{i}\right\}_{i \in \mathbb{N}}$ such that $h_{j}$ strongly converges to a nontrivial function $h_{\infty}$ in $\mathbb{P}_{0} X_{\lambda}$. However $\Gamma_{\lambda, 0}^{-1} \mathbb{P}_{0} \Pi_{1} \Gamma_{\lambda, \alpha_{i}}^{-1} \mathbb{P}_{0}^{\perp} f_{j}$ must converges to zero weakly in $X_{\lambda}$ since

$$
\begin{aligned}
\lim _{j \rightarrow \infty}<\mathbb{P}_{0} \Gamma_{\lambda, 0}^{-1} \Pi_{1} \Gamma_{\lambda, \alpha_{j}}^{-1} \mathbb{P}_{0}^{\perp} f_{j}, f>_{X_{\lambda}} & =\lim _{j \rightarrow \infty}<\Gamma_{\lambda, \alpha_{j}}^{-1} \mathbb{P}_{0}^{\perp} f_{j},\left(\Gamma_{\lambda, 0}^{-1} \Pi_{1}\right)^{*} \mathbb{P}_{0} f>_{X_{\lambda}} \\
& =0
\end{aligned}
$$

by the above claim. Here $\left(\Gamma_{\lambda, 0}^{-1} \Pi_{1}\right)^{*}$ is the (bounded) adjoint operator of $\Gamma_{\lambda, 0}^{-1} \Pi_{1}$. Hence $h_{\infty}=0$, which leads to a contradiction.

Now the proof of the proposition is completed.

4.2. Proof of Lemma 4.2. We are now in position to consider the equation $L_{\lambda, \alpha} h=$ $f$ for a given $f \in \mathbb{P}^{e} X_{\lambda}$. By the equality $\left(L_{\lambda, \alpha}-\lambda I\right) h=-\lambda h+f$, we substitute $-\lambda h+f$ for $\tilde{f}$ in (4.22) and (4.23). Then we have 
$(4.30)$

$$
\begin{aligned}
h_{S}= & -\lambda\left(-\mathcal{L}_{\lambda}\right)^{-\frac{1}{2}} \Gamma_{\lambda, 0}^{-1}\left(-\mathcal{L}_{\lambda}\right)^{-\frac{1}{2}} h_{S}+\lambda\left(-\mathcal{L}_{\lambda}\right)^{-\frac{1}{2}} \Gamma_{\lambda, 0}^{-1} \mathbb{P}_{0}\left(-\mathcal{L}_{\lambda}\right)^{-\frac{1}{2}} \mathcal{M} h_{S}^{\perp} \\
& -\left(-\mathcal{L}_{\lambda}\right)^{-\frac{1}{2}} \Gamma_{\lambda, 0}^{-1} \mathbb{P}_{0}\left(-\mathcal{L}_{\lambda}\right)^{-\frac{1}{2}} f \\
h_{S}^{\perp}= & -\lambda\left(-\mathcal{L}_{\lambda}\right)^{-\frac{1}{2}} \Gamma_{\lambda, \alpha}^{-1} \mathbb{P}_{0}^{\perp}\left(-\mathcal{L}_{\lambda}\right)^{-\frac{1}{2}} h_{S}^{\perp}+\lambda\left(-\mathcal{L}_{\lambda}\right)^{-\frac{1}{2}} \Gamma_{\lambda, \alpha}^{-1} \mathbb{P}_{0}^{\perp}\left(-\mathcal{L}_{\lambda}\right)^{-\frac{1}{2}} \mathcal{M} h \\
& -\left(-\mathcal{L}_{\lambda}\right)^{-\frac{1}{2}} \Gamma_{\lambda, \alpha}^{-1} \mathbb{P}_{0}^{\perp}\left(-\mathcal{L}_{\lambda}\right)^{-\frac{1}{2}} f
\end{aligned}
$$

By Proposition 4.1 we already have

$$
\begin{aligned}
\|h\|_{Y_{\lambda} \cap W_{\lambda}} & \leq \frac{C}{1-\lambda}\left\|\left(-\mathcal{L}_{\lambda}\right)^{-\frac{1}{2}}(-\lambda h+f)\right\|_{X_{\lambda}} \\
& \leq \frac{C \lambda}{(1-\lambda)^{\frac{3}{2}}}\|h\|_{X_{\lambda}}+\frac{C}{1-\lambda}\left\|\left(-\mathcal{L}_{\lambda}\right)^{-\frac{1}{2}} f\right\|_{X_{\lambda}} .
\end{aligned}
$$

So roughly speaking, it suffices to control $\|h\|_{X_{\lambda}}$ instead of $\|h\|_{Y_{\lambda} \cap W_{\lambda}}$. This is important for our purpose, since we can use the results of (4.25) and (4.26) in Proposition 4.2. By the estimate (4.25), we have good estimates for the non-radially symmetric part $h_{S}^{\perp}$ when $|\alpha|$ is large. Indeed, we have from (4.31) and (4.25),

$$
\begin{aligned}
\left\|h_{S}^{\perp}\right\|_{X_{\lambda}} & \leq \epsilon_{1}\left(\lambda\left\|\left(-\mathcal{L}_{\lambda}\right)^{-\frac{1}{2}} h_{S}^{\perp}\right\|_{X_{\lambda}}+\lambda\left\|\left(-\mathcal{L}_{\lambda}\right)^{-\frac{1}{2}} \mathcal{M} h\right\|_{X_{\lambda}}+\left\|\mathbb{P}_{0}^{\perp}\left(-\mathcal{L}_{\lambda}\right)^{-\frac{1}{2}} f\right\|_{X_{\lambda}}\right) \\
& \leq \epsilon_{1}\left(\frac{\lambda}{(1-\lambda)^{\frac{1}{2}}}\left\|h_{S}^{\perp}\right\| X_{X_{\lambda}}+\frac{C \lambda}{1-\lambda}\|h\|_{W_{\lambda}}+\left\|\mathbb{P}_{0}^{\perp}\left(-\mathcal{L}_{\lambda}\right)^{-\frac{1}{2}} f\right\| X_{X_{\lambda}}\right) .
\end{aligned}
$$

Thus by taking $\alpha$ large enough, it follows that

$$
\left\|h \frac{\perp}{S}\right\|_{X_{\lambda}} \leq \frac{C \epsilon_{1} \lambda}{1-\lambda}\|h\|_{W_{\lambda}}+2 \epsilon_{1}\left\|\mathbb{P}_{0}^{\perp}\left(-\mathcal{L}_{\lambda}\right)^{-\frac{1}{2}} f\right\|_{X_{\lambda}}
$$

from which we easily obtain the a priori estimates for the non-radially symmetric part $h_{S}^{\perp}$ in terms of $h_{S}$ and $f$.

Unfortunately we need more complicated arguments to control $h_{S}$ when $\lambda$ is not small, especially, when $\lambda \geq \frac{2}{3}$. Let us explain this difficulty. The second term in the right-hand side of (4.30) is a good term; we can estimate this term by combining (4.26) with the representation of (4.23). The difficulty appears when we deal with the first term in the right-hand side of (4.30). First we observe that

$$
\left\|\left(\mathcal{L}_{\lambda}+\lambda \mathcal{N}-\lambda I\right)^{-1} f\right\|_{X_{\lambda}} \leq \frac{1}{1-\frac{\lambda}{2}}\|f\|_{X_{\lambda}}
$$

Indeed, we have for $h=\left(\mathcal{L}_{\lambda}+\lambda \mathcal{N}-\lambda I\right)^{-1} f$, 


$$
\begin{aligned}
-<f, h>_{X_{\lambda}} & =<\left(-\mathcal{L}_{\lambda}-\lambda \mathcal{N}+\lambda I\right) h, h>_{X_{\lambda}} \\
& \geq(1-\lambda)\|h\|_{X_{\lambda}}^{2}-\lambda<\frac{x}{2} \cdot \nabla h, h>_{X_{\lambda}} \\
& =(1-\lambda)\|h\|_{X_{\lambda}}^{2}+\frac{\lambda}{2}\|h\|_{X_{\lambda}}^{2}+\frac{\lambda(1-\lambda)}{8}\|\| x \mid h \|_{X_{\lambda}}^{2} \\
& \geq\left(1-\frac{\lambda}{2}\right)\|h\|_{X_{\lambda}}^{2} .
\end{aligned}
$$

Here we used the fact $-\mathcal{L}_{\lambda} \geq 1-\lambda$ in $\mathbb{P}^{e} X_{\lambda}$ and the integration by parts. Thus we obtain (4.34). Since $-\left(-\mathcal{L}_{\lambda}\right)^{-\frac{1}{2}} \Gamma_{\lambda, 0}^{-1}\left(-\mathcal{L}_{\lambda}\right)^{-\frac{1}{2}}=\left(\mathcal{L}_{\lambda}+\lambda \mathcal{N}-\lambda I\right)^{-1}$, we have

$$
\left\|\lambda\left(-\mathcal{L}_{\lambda}\right)^{-\frac{1}{2}} \Gamma_{\lambda, 0}^{-1}\left(-\mathcal{L}_{\lambda}\right)^{-\frac{1}{2}} h_{S}\right\|_{X_{\lambda}} \leq \frac{\lambda}{1-\frac{\lambda}{2}}\left\|h_{S}\right\|_{X_{\lambda}}
$$

So the first term can be controlled at least when $\frac{\lambda}{1-\frac{\lambda}{2}}<1$, i.e., $\lambda<\frac{2}{3}$ since we get the contraction estimate in this case. In the case of $\lambda \geq \frac{2}{3}$ the above argument is no longer useful and we need further idea.

To overcome this difficulty, we use a cut-off technique as follows. Let $R>1$ and let $\chi_{R}$ be the characteristic function of the ball $\left\{x \in \mathbb{R}^{2}|| x \mid \leq R\right\}$. Then we define linear operators $\Phi_{R}$ and $\Phi_{R}^{c}$ as

$$
\begin{aligned}
\Phi_{R} f & =\chi_{R} f-\int_{\mathbb{R}^{2}} \chi_{R} f(x) d x G, \\
\Phi_{R}^{c} f & =\left(1-\chi_{R}\right) f-\int_{\mathbb{R}^{2}}\left(1-\chi_{R}\right) f(x) d x G .
\end{aligned}
$$

Here $G$ is the two-dimensional Gauss kernel. We see that

$$
\int_{\mathbb{R}^{2}} \Phi_{R} f d x=\int_{\mathbb{R}^{2}} \Phi_{R}^{c} f d x=0
$$

and if $\int_{\mathbb{R}^{2}} f(x) d x=0$, then $\Phi_{R} f+\Phi_{R}^{c} f=f$.

Especially, if $f \in \mathbb{P}_{0} X_{\lambda}$, so are $\Phi_{R} f$ and $\Phi_{R}^{c} f$. We also note that $\Phi_{R} f \in X_{0}$ by the definition. With these operators the equation (4.30) is written as

$$
h_{S}=h_{S}^{(1)}+h_{S}^{(2)}+F,
$$

where

$$
\begin{aligned}
& h_{S}^{(1)}=-\lambda\left(-\mathcal{L}_{\lambda}\right)^{-\frac{1}{2}} \Gamma_{\lambda, 0}^{-1}\left(-\mathcal{L}_{\lambda}\right)^{-\frac{1}{2}} \Phi_{R} h_{S}, \\
& h_{S}^{(2)}=-\lambda\left(-\mathcal{L}_{\lambda}\right)^{-\frac{1}{2}} \Gamma_{\lambda, 0}^{-1}\left(-\mathcal{L}_{\lambda}\right)^{-\frac{1}{2}} \Phi_{R}^{c} h_{S}, \\
& F=\lambda\left(-\mathcal{L}_{\lambda}\right)^{-\frac{1}{2}} \Gamma_{\lambda, 0}^{-1} \mathbb{P}_{0}\left(-\mathcal{L}_{\lambda}\right)^{-\frac{1}{2}} \mathcal{M} h_{S}^{\perp}-\left(-\mathcal{L}_{\lambda}\right)^{-\frac{1}{2}} \Gamma_{\lambda, 0}^{-1} \mathbb{P}_{0}\left(-\mathcal{L}_{\lambda}\right)^{-\frac{1}{2}} f .
\end{aligned}
$$

Then we have

Proposition 4.3. Let $\lambda \in[0,1)$. Then there is a positive $R_{1}=R_{1}(\lambda)>1$ such that the following estimate holds.

$$
\left\|h_{S}\right\|_{X_{\lambda}} \leq C e^{\frac{\lambda}{8} R_{1}^{2}}\|F\|_{X_{\lambda}} .
$$


Here the constant $C$ is independent of $\lambda \in[0,1), R_{1}$, and $\alpha$.

Proof of Proposition 4.3. We decompose $h_{S}$ into $\chi_{R} h_{S}$ and $\left(1-\chi_{R}\right) h_{S}$. For $\chi_{R} h_{S}$ we first estimate the norm in $X_{0}$ instead of $X_{\lambda}$. The key fact here is that $h_{S}^{(1)}$ belongs to $X_{0}$. Indeed, we recall that $\mathcal{L}$ is self-adjoint in $X_{0}$ and $-\mathcal{L} \geq 1$ in $\mathbb{P}_{0} X_{0}$. Especially, since $\Phi_{R} h_{S}$ belongs to $X_{0}$, the function $(\mathcal{L}-\lambda I)^{-1} \Phi_{R} h_{S}$ makes sense in $X_{0}$. So by the uniqueness of solutions to the equation $-\left(-\mathcal{L}_{\lambda}\right)^{-\frac{1}{2}}\left(I-\lambda \Pi_{2}+\lambda\left(-\mathcal{L}_{\lambda}\right)^{-1}\right)\left(-\mathcal{L}_{\lambda}\right)^{\frac{1}{2}} z_{1}=$ $(\mathcal{L}-\lambda I) z_{1}=z_{2}$ in $X_{\lambda}$ and $X_{0} \subset X_{\lambda}, h_{S}^{(1)}$ belongs to $\mathbb{P}_{0} X_{0}$.

We easily see that for any $z \in \mathbb{P}_{0} X_{0}$,

Thus we have

$$
\left\|(\mathcal{L}-\lambda I)^{-1} z\right\|_{X_{0}} \leq \frac{1}{1+\lambda}\|z\|_{X_{0}} .
$$

$$
\left\|h_{S}^{(1)}\right\|_{X_{0}} \leq \frac{\lambda}{1+\lambda}\left\|\Phi_{R} h_{S}\right\|_{X_{0}} \leq \frac{\lambda}{1+\lambda}\left(\left\|\chi_{R} h_{S}\right\|_{X_{0}}+\left|\int_{\mathbb{R}^{2}} \chi_{R} h_{S} d x\right|\right) .
$$

Since $\int_{\mathbb{R}^{2}} \chi_{R} h_{S} d x=-\int_{\mathbb{R}^{2}}\left(1-\chi_{R}\right) h_{S} d x$, we have by the Hölder inequality,

$$
\left|\int_{\mathbb{R}^{2}}\left(1-\chi_{R}\right) h_{S} d x\right| \leq\left\|G_{\lambda}^{\frac{1}{2}}\left(1-\chi_{R}\right)\right\|_{L^{2}}\left\|G_{\lambda}^{-\frac{1}{2}}\left(1-\chi_{R}\right) h_{S}\right\|_{L^{2}}=e^{-\frac{1-\lambda}{8} R^{2}}\left\|\left(1-\chi_{R}\right) h_{S}\right\|_{X_{\lambda}} .
$$

Hence we obtain

$$
\left\|h_{S}^{(1)}\right\|_{X_{0}} \leq \frac{\lambda}{1+\lambda}\left(\left\|\chi_{R} h_{S}\right\|_{X_{0}}+e^{-\frac{1-\lambda}{8} R^{2}}\left\|\left(1-\chi_{R}\right) h_{S}\right\|_{X_{\lambda}}\right) .
$$

Next we estimate $\chi_{R} h_{S}^{(2)}$. By the relation

$$
\begin{aligned}
\left\|\chi_{R} h_{S}^{(2)}\right\|_{X_{0}}=\left\|G^{-\frac{1}{2}} \chi_{R} h_{S}^{(2)}\right\|_{L^{2}} & \leq(1-\lambda)^{\frac{1}{2}} e^{\frac{\lambda}{8} R^{2}}\left\|G_{\lambda}^{-\frac{1}{2}} h_{S}^{(2)}\right\|_{L^{2}} \\
& =(1-\lambda)^{\frac{1}{2}} e^{\frac{\lambda}{8} R^{2}}\left\|h_{S}^{(2)}\right\|_{X_{\lambda}},
\end{aligned}
$$

we have from (4.34),

$$
\begin{aligned}
\left\|\chi_{R} h_{S}^{(2)}\right\|_{X_{0}} & \leq(1-\lambda)^{\frac{1}{2}} e^{\frac{\lambda}{8} R^{2}}\left\|h_{S}^{(2)}\right\|_{X_{\lambda}} \\
& \leq \frac{\lambda}{1-\frac{\lambda}{2}}(1-\lambda)^{\frac{1}{2}} e^{\frac{\lambda}{8} R^{2}}\left\|\Phi_{R}^{c} h_{S}\right\|_{X_{\lambda}} \\
& \leq \frac{2 \lambda}{2-\lambda}(1-\lambda)^{\frac{1}{2}} e^{\frac{\lambda}{8} R^{2}}\left(\left\|\left(1-\chi_{R}\right) h_{S}\right\|_{X_{\lambda}}+\|G\|_{X_{\lambda}}\left|\int_{\mathbb{R}^{2}}\left(1-\chi_{R}\right) h_{S} d x\right|\right) \\
& \leq 2 \lambda(1-\lambda)^{\frac{1}{2}} e^{\frac{\lambda}{8} R^{2}}\left(1+\left(1-\lambda^{2}\right)^{-\frac{1}{2}} e^{-\frac{1-\lambda}{8} R^{2}}\right)\left\|\left(1-\chi_{R}\right) h_{S}\right\|_{X_{\lambda}} .
\end{aligned}
$$

Collecting these above, we have

$$
\begin{aligned}
\left\|\chi_{R} h_{S}\right\|_{X_{0}} \leq & \frac{\lambda}{1+\lambda}\left(\left\|\chi_{R} h_{S}\right\|_{X_{0}}+e^{-\frac{1-\lambda}{8} R^{2}}\left\|\left(1-\chi_{R}\right) h_{S}\right\|_{X_{\lambda}}\right) \\
& +2 \lambda(1-\lambda)^{\frac{1}{2}} e^{\frac{\lambda}{8} R^{2}}\left(1+\left(1-\lambda^{2}\right)^{-\frac{1}{2}} e^{-\frac{1-\lambda}{8} R^{2}}\right)\left\|\left(1-\chi_{R}\right) h_{S}\right\|_{X_{\lambda}}+\left\|\chi_{R} F\right\|_{X_{0}},
\end{aligned}
$$

thus,

$\left\|\chi_{R} h_{S}\right\|_{X_{0}} \leq C \lambda\left(e^{-\frac{1}{8} R^{2}}+e^{-\frac{1-\lambda}{8} R^{2}}+(1-\lambda)^{\frac{1}{2}}\right) e^{\frac{\lambda}{8} R^{2}}\left\|\left(1-\chi_{R}\right) h_{S}\right\|_{X_{\lambda}}+2\left\|\chi_{R} F\right\|_{X_{0}}$, 
where $C$ is independent of $\lambda$ and $R$.

Next we consider $\left(1-\chi_{R}\right) h_{S}$. For the term $\left(1-\chi_{R}\right) h_{S}^{(1)}$, we have from Proposition 2.1 with $\lambda=0$,

$$
\begin{aligned}
\left\|\left(1-\chi_{R}\right) h_{S}^{(1)}\right\|_{X_{0}} & =\left(\int_{|x| \geq R} G^{-1}\left|h_{S}^{(1)}\right|^{2} d x\right)^{\frac{1}{2}} \\
& \leq R^{-1}\left(\int_{|x| \geq R} G^{-1}|x|^{2}\left|h_{S}^{(1)}\right|^{2} d x\right)^{\frac{1}{2}} \\
& \leq \lambda R^{-1}\left|\left\|x \mid(-\mathcal{L})^{-\frac{1}{2}}\left(I+\lambda(-\mathcal{L})^{-1}\right)^{-1}(-\mathcal{L})^{-\frac{1}{2}} \Phi_{R} h_{S}\right\|_{X_{0}}\right. \\
& \leq \frac{C \lambda}{R}\left\|\left(I+\lambda(-\mathcal{L})^{-1}\right)^{-1}(-\mathcal{L})^{-\frac{1}{2}} \Phi_{R} h_{S}\right\|_{X_{0}} \\
& \leq \frac{C \lambda}{R}\left\|\Phi_{R} h_{S}\right\|_{X_{0}} \\
& \leq \frac{C \lambda}{R}\left(\left\|\chi_{R} h_{S}\right\|_{X_{0}}+e^{-\frac{1-\lambda}{8} R^{2}}\left\|\left(1-\chi_{R}\right) h_{S}\right\|_{X_{\lambda}}\right) .
\end{aligned}
$$

Here we used the fact that $\left\|\left(I+\lambda(-\mathcal{L})^{-1}\right)^{-1}\right\|_{X_{0} \rightarrow X_{0}} \leq 1$. Thus we have

$$
\begin{aligned}
\left\|\left(1-\chi_{R}\right) h_{S}^{(1)}\right\|_{X_{\lambda}} & =\left(\int_{|x| \geq R} G_{\lambda}^{-1}\left|h_{S}^{(1)}\right|^{2} d x\right)^{\frac{1}{2}} \\
& \leq(1-\lambda)^{-\frac{1}{2}} e^{-\frac{\lambda}{8} R^{2}}\left(\int_{|x| \geq R} G^{-1}\left|h_{S}^{(1)}\right|^{2} d x\right)^{\frac{1}{2}} \\
& \leq \frac{C \lambda e^{-\frac{\lambda}{8} R^{2}}}{R(1-\lambda)^{\frac{1}{2}}}\left(\left\|\chi_{R} h_{S}\right\|_{X_{0}}+e^{-\frac{1-\lambda}{8} R^{2}}\left\|\left(1-\chi_{R}\right) h_{S}\right\|_{X_{\lambda}}\right) .
\end{aligned}
$$

Similarly from Proposition 2.1, we have for $\left(1-\chi_{R}\right) h_{S}^{(2)}$,

$$
\begin{aligned}
\left\|\left(1-\chi_{R}\right) h_{S}^{(2)}\right\|_{X_{\lambda}} & \leq \lambda R^{-1}\left\||x|\left(-\mathcal{L}_{\lambda}\right)^{-\frac{1}{2}} \Gamma_{\lambda, 0}^{-1}\left(-\mathcal{L}_{\lambda}\right)^{-\frac{1}{2}} \Phi_{R}^{c} h_{S}\right\|_{X_{\lambda}} \\
& \leq \frac{C \lambda}{R(1-\lambda)}\left\|\Gamma_{\lambda, 0}^{-1}\left(-\mathcal{L}_{\lambda}\right)^{-\frac{1}{2}} \Phi_{R}^{c} h_{S}\right\|_{X_{\lambda}} \\
& \leq \frac{C \lambda}{R(1-\lambda)^{\frac{3}{2}}}\left\|\Phi_{R}^{c} h_{S}\right\|_{X_{\lambda}} \\
& \leq \frac{C \lambda}{R(1-\lambda)^{\frac{3}{2}}}\left(1+\left(1-\lambda^{2}\right)^{-\frac{1}{2}} e^{-\frac{1-\lambda}{8} R^{2}}\right)\left\|\left(1-\chi_{R}\right) h_{S}\right\|_{X_{\lambda}} .
\end{aligned}
$$

Hence $\left(1-\chi_{R}\right) h_{S}$ is estimated as

$$
\begin{aligned}
& \left\|\left(1-\chi_{R}\right) h_{S}\right\|_{X_{\lambda}} \\
\leq & \left\|\left(1-\chi_{R}\right) h_{S}^{(1)}\right\|_{X_{\lambda}}+\left\|\left(1-\chi_{R}\right) h_{S}^{(2)}\right\|_{X_{\lambda}}+\left\|\left(1-\chi_{R}\right) F\right\|_{X_{\lambda}} \\
\leq & \frac{C \lambda e^{-\frac{\lambda}{8} R^{2}}}{R(1-\lambda)^{\frac{1}{2}}}\left(\left\|\chi_{R} h_{S}\right\|_{X_{0}}+e^{-\frac{1-\lambda}{8} R^{2}}\left\|\left(1-\chi_{R}\right) h_{S}\right\|_{X_{\lambda}}\right) \\
& +\frac{C \lambda}{R(1-\lambda)^{\frac{3}{2}}}\left(1+\left(1-\lambda^{2}\right)^{-\frac{1}{2}} e^{-\frac{1-\lambda}{8} R^{2}}\right)\left\|\left(1-\chi_{R}\right) h_{S}\right\|_{X_{\lambda}}+\left\|\left(1-\chi_{R}\right) F\right\|_{X_{\lambda}},
\end{aligned}
$$


that is,

$$
\left\|\left(1-\chi_{R}\right) h_{S}\right\|_{X_{\lambda}} \leq \frac{C \lambda e^{-\frac{\lambda}{8} R^{2}}}{R(1-\lambda)^{\frac{1}{2}}}\left\|\chi_{R} h_{S}\right\|_{X_{0}}+2\left\|\left(1-\chi_{R}\right) F\right\|_{X_{\lambda}}
$$

if $R$ is sufficiently large depending on $\lambda$.

Combining (4.43) and (4.44), we obtain

$$
\begin{aligned}
\left\|\chi_{R} h_{S}\right\|_{X_{0}} \leq & \frac{C \lambda^{2}}{R(1-\lambda)^{\frac{1}{2}}}\left(e^{-\frac{1}{8} R^{2}}+e^{-\frac{1-\lambda}{8} R^{2}}+(1-\lambda)^{\frac{1}{2}}\right)\left\|\chi_{R} h_{S}\right\|_{X_{0}} \\
& +C \lambda\left(e^{-\frac{1}{8} R^{2}}+e^{-\frac{1-\lambda}{8} R^{2}}+(1-\lambda)^{\frac{1}{2}}\right) e^{\frac{\lambda}{8} R^{2}}\left\|\left(1-\chi_{R}\right) F\right\|\left\|_{X_{\lambda}}+2\right\| \chi_{R} F \|_{X_{0}}
\end{aligned}
$$

thus, by the inequality $\left\|\chi_{R} F\right\|_{X_{0}} \leq(1-\lambda)^{\frac{1}{2}} e^{\frac{\lambda}{8} R^{2}}\left\|\chi_{R} F\right\|_{X_{\lambda}}$,

$$
\begin{gathered}
\left\|\chi_{R} h_{S}\right\|_{X_{0}} \leq C \lambda\left(e^{-\frac{1}{8} R^{2}}+e^{-\frac{1-\lambda}{8} R^{2}}+(1-\lambda)^{\frac{1}{2}}\right) e^{\frac{\lambda}{8} R^{2}}\left\|\left(1-\chi_{R}\right) F\right\|_{X_{\lambda}} \\
+C(1-\lambda)^{\frac{1}{2}} e^{\frac{\lambda}{8} R^{2}}\left\|\chi_{R} F\right\|_{X_{\lambda}},
\end{gathered}
$$

if $R$ is sufficiently large. Here $C$ is independent of $\lambda$ and $R$. From the estimates (4.44) and (4.45) we have

$$
\left\|\left(1-\chi_{R}\right) h_{S}\right\|_{X_{\lambda}} \leq \frac{C \lambda}{R(1-\lambda)^{\frac{1}{2}}}\left\|\chi_{R} F\right\|_{X_{\lambda}}+2\left\|\left(1-\chi_{R}\right) F\right\|_{X_{\lambda}} \leq C\|F\|_{X_{\lambda}}
$$

where $C$ can be taken as independent of $\lambda$ and $R$, if $R$ is sufficiently large.

Finally we see from (4.45) and (4.46),

$$
\begin{aligned}
\left\|h_{S}\right\|_{X_{\lambda}} & \leq\left\|\chi_{R} h_{S}\right\|_{X_{\lambda}}+\left\|\left(1-\chi_{R}\right) h_{S}\right\|_{X_{\lambda}} \\
& \leq(1-\lambda)^{-\frac{1}{2}}\left\|\chi_{R} h_{S}\right\|_{X_{0}}+\left\|\left(1-\chi_{R}\right) h_{S}\right\|_{X_{\lambda}} \\
& \leq C e^{\frac{\lambda}{8} R^{2}}\|F\|_{X_{\lambda}},
\end{aligned}
$$

if $R$ is sufficiently large. Here $C$ is independent of $\lambda$ and $R$. This completes the proof of Proposition 4.3.

Remark 4.1. From the above proof we have shown more precise estimate

$$
\left\|h_{S}\right\|_{X_{\lambda}} \leq C\left(e^{\frac{\lambda}{8} R^{2}}\left\|\chi_{R} F\right\|_{X_{\lambda}}+\left(1+\lambda e^{\frac{\lambda}{8} R^{2}}\right)\left\|\left(1-\chi_{R}\right) F\right\|_{X_{\lambda}}\right) .
$$

The number $R>>1$ is taken large enough to satisfy

$$
(1-\lambda)^{-\frac{3}{2}} R^{-1}<<1,
$$

since we have $(1-\lambda)^{-\frac{1}{2}} e^{-\frac{1-\lambda}{8} R^{2}}<<1$ in this case.

Proof of Lemma 4.2. In order to complete the proof of Lemma 4.2 we shall estimate the function $F$ defined by (4.40). By the representation (4.23) for $h \frac{\perp}{S}$, we observe that

$$
\begin{aligned}
F= & \left(-\mathcal{L}_{\lambda}\right)^{-\frac{1}{2}} \Gamma_{\lambda, 0}^{-1} \mathbb{P}_{0}\left(-\mathcal{L}_{\lambda}\right)^{-\frac{1}{2}} \mathcal{M}\left(-\mathcal{L}_{\lambda}\right)^{-\frac{1}{2}} \Gamma_{\lambda, \alpha}^{-1} \mathbb{P}_{0}^{\perp}\left\{-\lambda^{2}\left(-\mathcal{L}_{\lambda}\right)^{-\frac{1}{2}} h_{S}^{\perp}\right. \\
& \left.+\lambda^{2}\left(-\mathcal{L}_{\lambda}\right)^{-\frac{1}{2}} \mathcal{M} h-\lambda\left(-\mathcal{L}_{\lambda}\right)^{-\frac{1}{2}} f\right\}-\left(-\mathcal{L}_{\lambda}\right)^{-\frac{1}{2}} \Gamma_{\lambda, 0}^{-1} \mathbb{P}_{0}\left(-\mathcal{L}_{\lambda}\right)^{-\frac{1}{2}} f .
\end{aligned}
$$

Therefore we have from the estimate (4.26), 


$$
\begin{aligned}
\|F\|_{X_{\lambda}} \leq & \epsilon_{2}\left\|-\lambda^{2}\left(-\mathcal{L}_{\lambda}\right)^{-\frac{1}{2}} h_{S}^{\perp}+\lambda^{2}\left(-\mathcal{L}_{\lambda}\right)^{-\frac{1}{2}} \mathcal{M} h-\lambda \mathbb{P}_{0}^{\perp}\left(-\mathcal{L}_{\lambda}\right)^{-\frac{1}{2}} f\right\|_{X_{\lambda}} \\
& +(1-\lambda)^{-\frac{1}{2}}\left\|\mathbb{P}_{0}\left(-\mathcal{L}_{\lambda}\right)^{-\frac{1}{2}} f\right\|_{X_{\lambda}} \\
\leq & \frac{\epsilon_{2} \lambda^{2}}{1-\lambda}\|h\|_{W_{\lambda}}+\epsilon_{2} \lambda\left\|\mathbb{P}_{0}^{\perp}\left(-\mathcal{L}_{\lambda}\right)^{-\frac{1}{2}} f\right\|_{X_{\lambda}} \\
& +(1-\lambda)^{-\frac{1}{2}}\left\|\mathbb{P}_{0}\left(-\mathcal{L}_{\lambda}\right)^{-\frac{1}{2}} f\right\|_{X_{\lambda}}
\end{aligned}
$$

Combining this estimate with (4.41), we obtain

$$
\begin{aligned}
\left\|h_{S}\right\|_{X_{\lambda}} \leq & C e^{\frac{\lambda}{8} R^{2}}\left\{\frac{\epsilon_{2} \lambda^{2}}{1-\lambda}\|h\|_{W_{\lambda}}+\epsilon_{2} \lambda\left\|\mathbb{P}_{0}^{\perp}\left(-\mathcal{L}_{\lambda}\right)^{-\frac{1}{2}} f\right\|_{X_{\lambda}}\right. \\
& \left.+(1-\lambda)^{-\frac{1}{2}}\left\|\mathbb{P}_{0}\left(-\mathcal{L}_{\lambda}\right)^{-\frac{1}{2}} f\right\|_{X_{\lambda}}\right\} .
\end{aligned}
$$

Applying (4.33) and (4.48) for (4.32), we get the estimate

$$
\begin{aligned}
& \quad\|h\|_{Y_{\lambda} \cap W_{\lambda}} \\
& \leq \frac{C}{1-\lambda}\left\|\left(-\mathcal{L}_{\lambda}\right)^{-\frac{1}{2}} f\right\|_{X_{\lambda}}+\frac{C \lambda}{(1-\lambda)^{\frac{3}{2}}}\left(\epsilon_{1}\left\|\mathbb{P}_{0}^{\perp}\left(-\mathcal{L}_{\lambda}\right)^{-\frac{1}{2}} f\right\|_{X_{\lambda}}\right. \\
& \left.\quad+\epsilon_{2} \lambda e^{\frac{\lambda}{8} R^{2}}\left\|\mathbb{P}_{0}^{\perp}\left(-\mathcal{L}_{\lambda}\right)^{-\frac{1}{2}} f\right\|_{X_{\lambda}}+(1-\lambda)^{-\frac{1}{2}} e^{\frac{\lambda}{8} R^{2}}\left\|\mathbb{P}_{0}\left(-\mathcal{L}_{\lambda}\right)^{-\frac{1}{2}} f\right\|_{X_{\lambda}}\right) \\
& \leq \frac{C}{1-\lambda} e^{\frac{\lambda}{8} R^{2}}\left\|\left(-\mathcal{L}_{\lambda}\right)^{-\frac{1}{2}} f\right\|_{X_{\lambda}}
\end{aligned}
$$

if we take $|\alpha|$ sufficiently large. This estimate leads to the existence of the bounded inverse of $\mathcal{L}_{\lambda, \alpha}$ by the Fredholm alternative theorem. Now we have proved the estimate (4.9).

Collecting (4.49), (4.33), and (4.48), we obtain the estimates (4.10) and (4.11) for $\gamma=0$ as

$$
\begin{aligned}
& \left\|h_{S}^{\perp}\right\|_{X_{\lambda}} \leq \delta_{1}(|\alpha|, \lambda)\left(\lambda\left\|\left(-\mathcal{L}_{\lambda}\right)^{-\frac{1}{2}} f\right\|_{X_{\lambda}}+\left\|\mathbb{P}_{0}^{\perp}\left(-\mathcal{L}_{\lambda}\right)^{-\frac{1}{2}} f\right\|_{X_{\lambda}}\right) \\
& \left\|h_{S}\right\|_{X_{\lambda}} \leq \lambda \delta_{2}(|\alpha|, \lambda)\left\|\left(-\mathcal{L}_{\lambda}\right)^{-\frac{1}{2}} f\right\|_{X_{\lambda}}+\frac{C e^{\frac{\lambda}{8} R^{2}}}{(1-\lambda)^{\frac{1}{2}}}\left\|\mathbb{P}_{0}\left(-\mathcal{L}_{\lambda}\right)^{-\frac{1}{2}} f\right\|_{X_{\lambda}} .
\end{aligned}
$$

Here $\delta_{1}(|\alpha|, \lambda)$ and $\delta_{2}(|\alpha|, \lambda)$ satisfy

$$
\lim _{|\alpha| \rightarrow \infty} \delta_{i}(|\alpha|, \lambda)=0, i=1,2 .
$$

The estimates (4.10) and (4.11) for $\gamma>0$ are obtained in the similar way as above by using the representation (4.30), (4.31), the estimates (4.25), (4.26), and the above results $(4.49),(4.50),(4.51)$. We omit the details. The proof of Lemma 4.2 is now completed. 
4.3. Proof of Lemma 4.1. Let us prove Lemma 4.1. First we note that $\left(-\mathcal{L}_{\lambda}\right)^{-\frac{1}{2}}(\mathcal{L}+$ $\lambda \mathcal{M})(I-T)$ is bounded in $\mathbb{P}^{e} X_{\lambda}$ by Proposition 2.1 and Proposition 3.1. We also note that $(I-T) h_{2}=(I-T) \mathbb{P}_{0}^{\perp} h_{2}$. Then from the relation (4.6) and by Lemma 4.2, we get the estimates of $h_{2}$ in (4.6) such as

$$
\begin{aligned}
& \left\|h_{2}\right\|_{Y_{\lambda} \cap W_{\lambda}} \leq C\left\|\mathbb{P}_{0}^{\perp} h_{2}\right\|_{X_{\lambda}}+C\left\|\left(-\mathcal{L}_{\lambda}\right)^{-\frac{1}{2}} f_{1}\right\|_{X_{\lambda}}, \\
& \left\|\mathbb{P}_{0}^{\perp} h_{2}\right\|_{X_{\lambda}} \leq \delta\left\|\mathbb{P}_{0}^{\perp} h_{2}\right\|_{X_{\lambda}}+\delta\left(\left\|\mathbb{P}_{0}^{\perp}\left(-\mathcal{L}_{\lambda}\right)^{-\frac{1}{2}} f_{1}\right\|_{X_{\lambda}}+\lambda\left\|\left(-\mathcal{L}_{\lambda}\right)^{-\frac{1}{2}} f_{1}\right\|_{X_{\lambda}}\right), \\
& \left\|\mathbb{P}_{0} h_{2}\right\|_{X_{\lambda}} \leq C \lambda\left\|\mathbb{P}_{0}^{\perp} h_{2}\right\|_{X_{\lambda}}+C\left\|\mathbb{P}_{0}\left(-\mathcal{L}_{\lambda}\right)^{-\frac{1}{2}} f_{1}\right\|_{X_{\lambda}}+\lambda \delta\left\|\left(-\mathcal{L}_{\lambda}\right)^{-\frac{1}{2}} f_{1}\right\|_{X_{\lambda}},
\end{aligned}
$$

where $C$ depends only on $\lambda$, and $\delta$ is sufficiently small by taking $|\alpha|$ large enough. For $h_{1}=T h_{2}$ we see that $\mathbb{P}_{0} h_{1}=\mathbb{P}_{0} h_{2}$, and $\mathbb{P}_{0}^{\perp} h_{1}=\mathbb{P}_{0}^{\perp} h_{2}+(T-I) \mathbb{P}_{0}^{\perp} h_{2}$ hold. Now Lemma 4.1 easily follows when $\gamma=0$, and then, also when $\gamma \in(0,1)$. This completes the proof of Lemma 4.1.

\section{Construction of asymmetric Burgers vortices for large CIRCULATION NUMBERS}

Based on the results for the linearization established in the previous section, we can construct solutions to (1.22) by perturbation arguments. Our aim is to solve the equation

$$
w=(\mathcal{L}+\lambda \mathcal{M}-\alpha \Lambda)^{-1}\left(B(w, w)+\lambda \Lambda_{w_{\infty}} w+\lambda f_{\lambda}\right),
$$

where $\Lambda_{w_{\infty}}$ and $f_{\lambda}$ are given by (1.17) and (1.23). Since we already have the estimates for $(\mathcal{L}+\lambda \mathcal{M}-\alpha \Lambda)^{-1}$ by Lemma 4.1 , the argument to solve (5.1) is just parallel to $[14$, section 4]. So we only give the sketch of the proof here.

To apply Lemma 4.1 we decompose (5.1) into the radially symmetric part and the non-radially symmetric part. That is, we construct a solution of the form

$$
w=w_{S}+w_{S^{\perp}}, w_{S} \in Y_{\lambda} \cap W_{\lambda} \cap \mathbb{P}_{0} X_{\lambda}, w_{S^{\perp}} \in Y_{\lambda} \cap W_{\lambda} \cap \oplus_{n \in \mathbb{Z}, n \neq 0} \mathbb{P}_{2 n} X_{\lambda} .
$$

Then we see

$$
\begin{aligned}
B(w, w) & =B\left(w_{S}, w_{S^{\perp}}\right)+B\left(w_{S^{\perp}}, w_{S}\right)+B\left(w_{S^{\perp}}, w_{S^{\perp}}\right) \\
& =\Lambda_{w_{S}} w_{S^{\perp}}+\frac{1}{2} \Lambda_{w_{S}} w_{S^{\perp}}, \\
\Lambda_{w_{\infty}} w & =\Lambda_{w_{\infty}} w_{S}+\Lambda_{w_{\infty}} w_{S^{\perp}} .
\end{aligned}
$$

Fix $\gamma \in(0,1)$. We identify $D\left(A_{\lambda}^{\gamma}\right)$ in $\mathbb{P}^{e} X_{\lambda}$ with $D_{S} \times D_{S^{\perp}}$, where

$$
D_{S}=D\left(A_{\lambda}^{\gamma}\right) \cap \mathbb{P}_{0} X_{\lambda}, D_{S^{\perp}}=D\left(A_{\lambda}^{\gamma}\right) \cap \oplus_{n \in \mathbb{Z}, n \neq 0} \mathbb{P}_{2 n} X_{\lambda} .
$$

For $(f, h) \in D_{S} \times D_{S^{\perp}}$, we define the mappings

$$
\begin{aligned}
H_{1}(f, h) & =(\mathcal{L}-\alpha \Lambda+\lambda \mathcal{M})^{-1} \Lambda_{f} h, \\
H_{2}(f, h) & =\frac{1}{2}(\mathcal{L}-\alpha \Lambda+\lambda \mathcal{M})^{-1} \Lambda_{h} h, \\
H_{3}(f, h) & =\lambda(\mathcal{L}-\alpha \Lambda+\lambda \mathcal{M})^{-1} \Lambda_{w_{\infty}} f, \\
H_{4}(f, h) & =\lambda(\mathcal{L}-\alpha \Lambda+\lambda \mathcal{M})^{-1} \Lambda_{w_{\infty}} h, \\
F_{\lambda, \alpha} & =\lambda(\mathcal{L}-\alpha \Lambda+\lambda \mathcal{M})^{-1} f_{\lambda},
\end{aligned}
$$


and

$$
H_{\lambda, \alpha}(f, h)=\sum_{i=1}^{4} H_{i}(f, h)+F_{\lambda, \alpha} .
$$

Since $f_{\lambda} \in D_{S^{\perp}}$, we observe from (4.2) and (4.3) that $\lim _{|\alpha| \rightarrow \infty}\left\|F_{\lambda, \alpha}\right\|_{D\left(A_{\lambda}^{\gamma}\right)}=0$ (in fact, we have a better estimate; see (5.13) below).

The map $H_{\lambda, \alpha}$ is compact. Indeed, from (4.1) in Lemma 4.1 we see that $H_{\lambda, \alpha}$ maps $D_{S} \times D_{S^{\perp}}$ into $Y_{\lambda} \cap W_{\lambda} \cap \mathbb{P}^{e} X_{\lambda}$. Now we set the map $\Psi_{\lambda, \alpha}$ on $D_{S} \times D_{S^{\perp}}$ as

$$
\Psi_{\lambda, \alpha}(f, h)=\left(\mathbb{P}_{0} H_{\lambda, \alpha}(f, h), \mathbb{P}_{0}^{\perp} H_{\lambda, \alpha}(f, h)\right),
$$

where $\mathbb{P}_{0}^{\perp}=I-\mathbb{P}_{0}$. Arguing as in [14, Proposition 4.1], we can show that for any $\kappa_{1}>0$, there are $\kappa_{2} \geq \kappa_{1}$ and $R=R\left(\lambda, \kappa_{1}, \kappa_{2}\right)>>1$ such that $\Psi_{\lambda, \alpha}$ has a unique fixed point in the closed subset

$$
X_{\kappa_{2}, \kappa_{1}}=\left\{(f, h) \in D_{S} \times D_{S^{\perp}} \mid\|f\|_{D\left(A_{\lambda}^{\gamma}\right)} \leq \kappa_{2},\|h\|_{D\left(A_{\lambda}^{\gamma}\right)} \leq \kappa_{1}\right\},
$$

if $|\alpha| \geq R$. Indeed, from (4.2) in Lemma 4.1 and (2.23) we have for $\left(f_{i}, h_{i}\right) \in$ $X_{\kappa_{2}, \kappa_{1}}, i=1,2$,

$$
\begin{aligned}
& \left\|\mathbb{P}_{0}^{\perp} H_{\lambda, \alpha}\left(f_{1}, h_{1}\right)-\mathbb{P}_{0}^{\perp} H_{\lambda, \alpha}\left(f_{2}, h_{2}\right)\right\|_{D\left(A_{\lambda}^{\gamma}\right)} \\
\leq & C \delta_{1}\left(\kappa_{1}+\kappa_{2}\right)\left(\left\|f_{1}-f_{2}\right\|_{D\left(A_{\lambda}^{\gamma}\right)}+\left\|h_{1}-h_{2}\right\|_{D\left(A_{\lambda}^{\gamma}\right)}\right),
\end{aligned}
$$

where $\delta_{1}$ is sufficiently small if $|\alpha|$ is large enough. Similarly, we have from (4.3) and $(2.23)$,

$$
\begin{aligned}
& \left\|\mathbb{P}_{0} H_{\lambda, \alpha}\left(f_{1}, h_{1}\right)-\mathbb{P}_{0} H_{\lambda, \alpha}\left(f_{2}, h_{2}\right)\right\|_{D\left(A_{\lambda}^{\gamma}\right)} \\
\leq & C\left(\kappa_{1}+1\right)\left\|h_{1}-h_{2}\right\|_{D\left(A_{\lambda}^{\gamma}\right)}+C \delta_{2}\left(\kappa_{1}+\kappa_{2}+1\right)\left(\left\|f_{1}-f_{2}\right\|_{D\left(A_{\lambda}^{\gamma}\right)}+\left\|h_{1}-h_{2}\right\|_{D\left(A_{\lambda}^{\gamma}\right)}\right),
\end{aligned}
$$

where $\delta_{2}$ is sufficiently small if $|\alpha|$ is large enough. So $\Psi_{\lambda, \alpha}$ is completely continuous. Since $\left\|\Psi_{\lambda, \alpha}(f, h)\right\|_{D\left(A_{\lambda}^{\gamma}\right)} \leq\left\|\Psi_{\lambda, \alpha}(f, h)-\Psi_{\lambda, \alpha}(0,0)\right\|_{D\left(A_{\lambda}^{\gamma}\right)}+\left\|F_{\lambda, \alpha}\right\|_{D\left(A_{\lambda}^{\gamma}\right)}$ and $\lim _{|\alpha| \rightarrow \infty}\left\|F_{\lambda, \alpha}\right\|_{D\left(A_{\lambda}^{\gamma}\right)}=0$, we see from the above estimates that $\Psi_{\lambda, \alpha}$ maps $X_{\kappa_{2}, \kappa_{1}}$ into $X_{\kappa_{2}, \kappa_{1}}$ for any $\kappa_{1}>0$ and suitable $\kappa_{2} \geq \kappa_{1}$ and $|\alpha|>>1$. Thus there is a fixed point in $X_{\kappa_{2}, \kappa_{1}}$ by the Schauder fixed point theorem. The uniqueness of fixed points in $X_{\kappa_{2}, \kappa_{1}}$ also follows from (5.10) and (5.11). This proves Theorem 1.2 and Theorem 1.3 except for the asymptotic estimate (1.19).

Let $\left(w_{S}, w_{S^{\perp}}\right)$ be the fixed point of $\Psi_{\lambda, \alpha}$ in $X_{\kappa_{2}, \kappa_{1}}$. Then from (5.10), (5.11), and

$$
\begin{aligned}
\left\|\left(w_{S}, w_{S^{\perp}}\right)\right\|_{D\left(A_{\lambda}^{\gamma}\right)} & =\left\|\Psi_{\lambda, \alpha}\left(w_{S}, w_{S^{\perp}}\right)\right\|_{D\left(A_{\lambda}^{\gamma}\right)} \\
& \leq\left\|\Psi_{\lambda, \alpha}\left(w_{S}, w_{S^{\perp}}\right)-\Psi_{\lambda, \alpha}(0,0)\right\|_{D\left(A_{\lambda}^{\gamma}\right)}+\left\|F_{\lambda, \alpha}\right\|_{D\left(A_{\lambda}^{\gamma}\right)},
\end{aligned}
$$

we get

$$
\left\|\left(w_{S}, w_{S^{\perp}}\right)\right\|_{D\left(A_{\lambda}^{\gamma}\right)} \leq C|| F_{\lambda, \alpha} \|_{D\left(A_{\lambda}^{\gamma}\right)} .
$$

Moreover, we have from (4.1) and (2.23),

$$
\left\|\left(w_{S}, w_{S^{\perp}}\right)\right\|_{Y_{\lambda} \cap W_{\lambda}} \leq C\left(\left\|\left(w_{S}, w_{S^{\perp}}\right)\right\|_{D\left(A_{\lambda}^{\gamma}\right)}+\left\|\left(w_{S}, w_{S^{\perp}}\right)\right\|_{D\left(A_{\lambda}^{\gamma}\right)}^{2}+\left\|F_{\lambda, \alpha}\right\|_{Y_{\lambda} \cap W_{\lambda}}\right),
$$

where $C$ depends only on $\lambda$. Combining these above, we obtain

$$
\left\|\left(w_{S}, w_{S^{\perp}}\right)\right\|_{Y_{\lambda} \cap W_{\lambda}} \leq C\left\|F_{\lambda, \alpha}\right\|_{Y_{\lambda} \cap W_{\lambda}} .
$$


Thus it suffices to show

$$
\left\|F_{\lambda, \alpha}\right\|_{Y_{\lambda} \cap W_{\lambda}} \leq \frac{C \lambda}{1+|\alpha|}
$$

for $|\alpha|>>1$. This estimate is obtained from the fact that there is a function $h_{\lambda} \in Y_{\lambda} \cap W_{\lambda}$ such that $f_{\lambda}=\Lambda h_{\lambda}$; see [7, Proposition 3.4] or [14, Proposition 5.1]. Indeed, since we have the relation

$$
\begin{aligned}
F_{\lambda, \alpha} & =\lambda(\mathcal{L}+\lambda \mathcal{M}-\alpha \Lambda)^{-1} f_{\lambda} \\
& =\lambda(\mathcal{L}+\lambda \mathcal{M}-\alpha \Lambda)^{-1} \Lambda h_{\lambda} \\
& =-\frac{\lambda}{\alpha}(\mathcal{L}+\lambda \mathcal{M}-\alpha \Lambda)^{-1}(\mathcal{L}+\lambda \mathcal{M}-\alpha \Lambda-\mathcal{L}-\lambda \mathcal{M}) h_{\lambda} \\
& =\frac{\lambda}{\alpha} h_{\lambda}+\frac{\lambda}{\alpha}(\mathcal{L}+\lambda \mathcal{M}-\alpha \Lambda)^{-1}(\mathcal{L}+\lambda \mathcal{M}) h_{\lambda},
\end{aligned}
$$

we get (5.13) from (4.1). This completes the proof of Theorem 1.2.

\section{Existence of ASymmetric Burgers vortices in General CASES}

In this section we prove the existence of the asymmetric Burgers vortices for general asymmetry parameters $\lambda \in[0,1)$ and circulation numbers $\alpha \in \mathbb{R}$. Let us recall the equations for the asymmetric Burgers vortices:

$\left(B_{\lambda, \alpha}\right)$

$$
\left\{\begin{array}{l}
(\mathcal{L}+\lambda \mathcal{M}) \omega-(K * \omega, \nabla) \omega=0, x \in \mathbb{R}^{2}, \\
\int_{\mathbb{R}^{2}} \omega(x) d x=\alpha .
\end{array}\right.
$$

Then solutions to $\left(B_{\lambda, \alpha}\right)$ are constructed as solutions to

$$
\omega=-(\mathcal{L}+\lambda \mathcal{M}-I)^{-1} \omega+(\mathcal{L}+\lambda \mathcal{M}-I)^{-1} \nabla \cdot(\omega K * \omega) .
$$

Let

$$
\mathcal{G}_{\lambda}(x)=\frac{\sqrt{1-\lambda^{2}}}{4 \pi} e^{-\frac{1+\lambda}{4} x_{1}^{2}-\frac{1-\lambda}{4} x_{2}^{2}} .
$$

Then by the relation $-(\mathcal{L}+\lambda \mathcal{M}-I)^{-1} \mathcal{G}_{\lambda}=\mathcal{G}_{\lambda},(6.1)$ is equivalent to the equation for $w=\omega-\alpha \mathcal{G}_{\lambda}$ :

$$
\begin{aligned}
w= & -(\mathcal{L}+\lambda \mathcal{M}-I)^{-1} w+(\mathcal{L}+\lambda \mathcal{M}-I)^{-1} \nabla \cdot(w K * w) \\
& +(\mathcal{L}+\lambda \mathcal{M}-I)^{-1}\left(\alpha \Lambda_{\mathcal{G}_{\lambda}} w+\alpha^{2}\left(K * \mathcal{G}_{\lambda}, \nabla\right) \mathcal{G}_{\lambda}\right) .
\end{aligned}
$$

We set the right hand side of $(6.3)$ as $\Psi(w)$. So our aim is to look for a fixed point of $\Psi$ in $X_{\lambda}$ by using the Schauder fixed point theorem, which gives Theorem 1.1. We first observe that $\Psi$ is a completely continuous mapping defined on $D\left(A_{\lambda}^{\gamma}\right)$ where $A_{\lambda}=\left(-\mathcal{L}_{\lambda}\right)^{\frac{1}{2}}$ and $\gamma \in(0,1)$. Indeed, similar arguments as in the previous sections give the estimates

$$
\begin{aligned}
& \left\|(\mathcal{L}+\lambda \mathcal{M}-I)^{-1} f_{1}\right\|_{Y_{\lambda} \cap W_{\lambda}} \leq C\left\|f_{1}\right\|_{X_{\lambda}} \\
& \left\|(\mathcal{L}+\lambda \mathcal{M}-I)^{-1} \nabla \cdot\left(f_{2} K * f_{3}\right)\right\|_{Y_{\lambda} \cap W_{\lambda}} \leq C\left\|f_{2}\right\|_{X_{\lambda}}\left\|K * f_{3}\right\|_{L^{\infty}} \\
& \left\|K * f_{3}\right\|_{L^{\infty}} \leq C\left\|f_{3}\right\|_{L^{1}}^{\frac{1}{2}}\left\|f_{3}\right\|_{L^{\infty}}^{\frac{1}{2}} \\
& \left\|K * f_{3}\right\|_{L^{\infty}} \leq C\left\|f_{3}\right\|_{D\left(A_{\lambda}^{\gamma}\right)},
\end{aligned}
$$


for any $f_{i} \in D\left(A_{\lambda}^{\gamma}\right)$ and $\gamma \in(0,1)$. Indeed, (6.4) and (6.5) are obtained by the argument as in the proof of Proposition 4.1, and (6.6) and (6.7) are established by the Hardy-Littlewood-Sobolev inequalities and the interpolation arguments. We omit the details here. Hence $\Psi$ is completely continuous and it suffices to show that the set

$$
\left\{w \in D\left(A_{\lambda}^{\gamma}\right) \mid w=\delta \Psi(w), \delta \in[0,1]\right\}
$$

is bounded. For simplicity we always take $\gamma=\frac{1}{2}$. Without loss of generality, we may assume that $\alpha$ is positive. Let $w=\delta \Psi(w)$. Then from (6.4)-(6.7), and the interpolation inequality $\|w\|_{D\left(A_{\lambda}^{\frac{1}{2}}\right)} \leq C\|w\|_{Y_{\lambda} \cap W_{\lambda}}^{\frac{1}{2}}\|w\|_{X_{\lambda}}^{\frac{1}{2}}$, it is not difficult to obtain the estimate

$$
\|w\|_{Y_{\lambda} \cap W_{\lambda}} \leq C_{1}\left(\|w\|_{X_{\lambda}}+\|w\|_{X_{\lambda}}^{3}\right)+C_{2}
$$

where $C_{1}$ and $C_{2}$ depend only on $\lambda$ and $\alpha$. From now on, we estimate $\|w\|_{X_{\lambda}}$. If $w$ satisfies $w=\delta \Psi(w)$, then $\omega=w+\alpha \mathcal{G}_{\lambda}$ solves the equation

$$
\left\{\begin{array}{l}
(\mathcal{L}+\lambda \mathcal{M}-(1-\delta) I) \omega=-\alpha(1-\delta) \mathcal{G}_{\lambda}+\delta(K * \omega, \nabla) \omega, x \in \mathbb{R}^{2}, \\
\int_{\mathbb{R}^{2}} \omega(x) d x=\alpha .
\end{array}\right.
$$

First we consider the case $\delta<1$. Let us introduce the scaling variables $\xi \in \mathbb{R}^{2}$ and $\tau \geq 1$ as

$$
x=\frac{\xi}{\sqrt{\tau}} .
$$

Set $v(\tau, \xi)=\tau^{-1} \omega\left(\frac{\xi}{\sqrt{\tau}}\right)$. By direct calculations, we get the equation for $v$ :

$\left\{\begin{array}{l}\partial_{\tau} v-\Delta v-\frac{\lambda}{\sqrt{\tau}} \mathcal{M} v+\frac{(1-\delta)}{\tau} v=\frac{\alpha(1-\delta)}{\tau^{2}} \mathcal{G}_{\lambda}\left(\frac{\xi}{\sqrt{\tau}}\right)-\delta(K * v, \nabla) v, \tau>1, \quad \xi \in \mathbb{R}^{2}, \\ \left.v\right|_{\tau=1}=\omega .\end{array}\right.$

Now we claim that $\left(\|\omega\|_{L^{1}}=\right)\|v(\tau)\|_{L^{1}}=\alpha$ and so $\omega$ is nonnegative. Indeed, if we set $\phi_{\epsilon}(r)=\left(r^{2}+\epsilon^{2}\right)^{\frac{1}{2}}-\epsilon$ for $r, \epsilon>0$, then $0 \leq \phi_{\epsilon}(r) \leq r$ and we have

$$
\begin{aligned}
\partial_{\tau} \int_{\mathbb{R}^{2}} \phi_{\epsilon}(v(\tau, \xi)) d \xi= & -\int_{\mathbb{R}^{2}} \phi_{\epsilon}^{\prime \prime}(v)|\nabla v(\tau, \xi)|^{2} d \xi-\frac{(1-\delta)}{\tau} \int_{\mathbb{R}^{2}} \phi_{\epsilon}^{\prime}(v) v(\tau, \xi) d \xi \\
& +\frac{\alpha(1-\delta)}{\tau^{2}} \int_{\mathbb{R}^{2}} \phi_{\epsilon}^{\prime}(v) \mathcal{G}_{\lambda}\left(\frac{\xi}{\sqrt{\tau}}\right) d \xi .
\end{aligned}
$$

Here we used the fact that if $u(\tau)$ is a given vector field satisfying $\nabla \cdot u(\tau)=0$, then

$$
\int_{\mathbb{R}^{2}} \phi_{\epsilon}^{\prime}(v)(u, \nabla) v(\tau, \xi) d \xi=\int_{\mathbb{R}^{2}}(u, \nabla) \phi_{\epsilon}(v(\tau, \xi)) d \xi=\int_{\mathbb{R}^{2}} \nabla \cdot\left(u \phi_{\epsilon}(v(\tau, \xi)) d \xi=0 .\right.
$$

Hence from $\phi_{\epsilon}^{\prime \prime}(v)>0$, it follows that

$$
\partial_{\tau} \int_{\mathbb{R}^{2}} \phi_{\epsilon}(v(\tau, \xi)) d \xi \leq-\frac{(1-\delta)}{\tau} \int_{\mathbb{R}^{2}} \phi_{\epsilon}^{\prime}(v) v(\tau, \xi) d \xi+\frac{\alpha(1-\delta)}{\tau} .
$$

So

$$
\int_{\mathbb{R}^{2}} \phi_{\epsilon}(v(\tau, \xi)) d \xi \leq \int_{\mathbb{R}^{2}} \phi_{\epsilon}(\omega(\xi)) d \xi-(1-\delta) \int_{1}^{\tau} s^{-1} \int_{\mathbb{R}^{2}} \phi_{\epsilon}^{\prime}(v) v(s, \xi) d \xi d s+\alpha(1-\delta) \log \tau \text {. }
$$


Tending $\epsilon$ to 0 , we see from the Lebesgue convergence theorem,

$$
\|v(\tau)\|_{L^{1}} \leq\|\omega\|_{L^{1}}-(1-\delta) \int_{1}^{\tau} s^{-1}\|v(s)\|_{L^{1}} d s+\alpha(1-\delta) \log \tau .
$$

Thus from the Gronwall inequality, we have

$$
\|v(\tau)\|_{L^{1}} \leq \tau^{-1+\delta}\|\omega\|_{L^{1}}+\alpha\left(1-\tau^{-1+\delta}\right) .
$$

But since $\|v(\tau)\|_{L^{1}}=\|\omega\|_{L^{1}}$, the above inequality implies $\|v(\tau)\|_{L^{1}} \leq \alpha$ when $\delta<1$. Thus we have $\|v(\tau)\|_{L^{1}}=\alpha$ and especially, $v(\tau)$ is nonnegative. Also in the case $\delta=1$, we can get the same result of $\|\omega\|_{L^{1}}=\|v(\tau)\|_{L^{1}}=\alpha$ by the maximum principle; see Remark 4 in [6, Section 4]. Next we consider the evolution operator $\Gamma(\tau, s)$ associated with $\partial_{\tau} v-\Delta v-\frac{\lambda}{\sqrt{\tau}} \mathcal{M} v+\frac{(1-\delta)}{\tau} v+\delta(K * v, \nabla) v$. Since $-\frac{\lambda}{\sqrt{\tau}} \mathcal{M} v+\delta(K * v, \nabla) v$ is a convective term by the divergence free vector fields, by the results of Carlen-Loss [2, Theorem 1] we can get the $L^{p}-L^{q}$ estimates which do not depend on the terms $\frac{\lambda}{\sqrt{\tau}} \mathcal{M} v$ and $(K * v, \nabla) v$. More precisely, we have

$$
\|\Gamma(\tau, 1) f\|_{L^{p}} \leq \frac{C}{(\tau-1)^{\frac{1}{q}-\frac{1}{p}}}\|f\|_{L^{q}}, \tau>1,1 \leq q \leq p \leq \infty .
$$

Here $C$ is a numerical constant. Since $v(\tau)$ is written as

$$
v(\tau)=\Gamma(\tau, 1) \omega+\alpha(1-\delta) \int_{1}^{\tau} \Gamma(\tau, s) s^{-2} \mathcal{G}_{\lambda}\left(\frac{\dot{-}}{s}\right) d s
$$

we get

$$
\begin{aligned}
\|v(\tau)\|_{L^{p}} & \leq \frac{C}{(\tau-1)^{1-\frac{1}{p}}}\|\omega\|_{L^{1}}+\alpha(1-\delta) \int_{1}^{\tau} s^{-2+\frac{1}{p}} d s \\
& \leq \frac{C}{(\tau-1)^{1-\frac{1}{p}}} \alpha+\alpha(1-\delta) C_{p}, 1<p \leq \infty .
\end{aligned}
$$

Then taking $\tau=2$, we have $\|\omega\|_{L^{p}}=2^{1-\frac{1}{p}}\|v(2)\|_{L^{p}} \leq C \alpha$ where $C$ depends only on $p$. Hence we have $\|w\|_{L^{p}} \leq C_{p} \alpha$ for any $1 \leq p \leq \infty$. This a priori $L^{p}$ estimates for solutions of $w=\delta \Psi(w)$ lead to the a priori estimates in $X_{\lambda}$. To prove this, we estimate $\left\|\chi_{R} w\right\|_{X_{\lambda}}$ where $\chi_{R}$ is a cut off function such that $\chi_{R}(x)=0$ if $|x| \leq R$ and $\chi_{R}(x)=1$ otherwise. Let us give the estimate for the nonlinear term $(\mathcal{L}+\lambda \mathcal{M}-I)^{-1} \nabla \cdot(w K * w)$ in the definition of $\Psi(w)$. From (6.5), (6.7), and the a priori $L^{p}$ estimates for $w$, we have

$$
\begin{aligned}
\left\|\chi_{R}(\mathcal{L}+\lambda \mathcal{M}-I)^{-1} \nabla \cdot(w K * w)\right\|_{X_{\lambda}} & \leq R^{-1}\left|\left\|x \mid(\mathcal{L}+\lambda \mathcal{M}-I)^{-1} \nabla \cdot(w K * w)\right\|_{X_{\lambda}}\right. \\
& \leq C R^{-1}\|w\|\left\|_{X_{\lambda}}\right\| K * w \|_{L^{\infty}} \\
& \leq C R^{-1}\left(\left\|\left(1-\chi_{R}\right) w\right\|_{X_{\lambda}}+\left\|\chi_{R} w\right\|_{X_{\lambda}}\right) \alpha \\
& \leq C_{1} \alpha R^{-1}\left\|\chi_{R} w\right\|_{X_{\lambda}}+C_{2}
\end{aligned}
$$

Here $C_{1}$ depends only on $\lambda$, and $C_{2}$ depends only on $\alpha, \lambda$, and $R$. Then from similar arguments, we have

$$
\left\|\chi_{R} w\right\|_{X_{\lambda}}=\delta\left\|\chi_{R} \Psi(w)\right\|_{X_{\lambda}} \leq C_{1}^{\prime} \alpha R^{-1}\left\|\chi_{R} w\right\|_{X_{\lambda}}+C_{2}^{\prime},
$$

where $C_{1}^{\prime}$ depends only on $\lambda$, and $C_{2}^{\prime}$ depends only on $\alpha, \lambda$, and $R$. So if we take $R$ large enough to satisfy $C_{1}^{\prime} \alpha R^{-1} \leq \frac{1}{2}$, then we obtain the a priori estimate

$$
\left\|\chi_{R} w\right\|_{X_{\lambda}} \leq 2 C_{2}^{\prime} \text {. }
$$


Note that the choice of $R$ depends only on $\alpha$ and $\lambda$. Combining (6.16) with the a

priori estimates in $L^{p}$ and (6.9), we get the desired a priori estimate in $D\left(A_{\lambda}^{\frac{1}{2}}\right)$ for all fixed points of $w=\delta \Psi(w), \delta \in[0,1]$. The proof is now complete.

We conclude this section by giving a remark on the stability of the Burgers vortices which is not discussed in this paper.

Remark 6.1 (Stability of Burgers vortices). Since the axisymmetric Burgers vortex $\alpha G$ gives the nontrivial exact solution to three dimensional Navier-Stokes equations, its stability problem has attracted many researchers. In Giga-Kambe [9] it is proved that if the $L^{1}$-norm of initial data is sufficiently small, then the solution of the nonstationary equation associated with $\left(B_{\lambda, \alpha}\right)$ with $\lambda=0$ converges to $\alpha G$ where $\alpha$ is the total circulation of initial vorticity (note that the total circulation is conserved under the equation $\left.\left(B_{\lambda, \alpha}\right)\right)$. Their result is extended by Carpio [3] and Giga-Giga [8] in which the global stability of the axisymmetric Burgers vortex (with respect to two dimensional perturbations) is obtained when the vortex Reynolds number is sufficiently small. Although the global stability for not small vortex Reynolds numbers had remained open for years, the affirmative answer is given by Gallay-Wayne [5]. The rate of convergence is also discussed there. As indicated by [15], it is important to consider the influence on the stability by a fast rotation $|\alpha|>>1$. In [13] the spectrum of $\mathcal{L}-\alpha \Lambda_{G}$ in $X_{0}$ is studied and the rate of convergence to axisymmetric Burgers vortices is improved when the vortex Reynolds number is sufficiently large.

As for the asymmetric Burgers vortices, as far as the author knows, the mathematical understanding of their stability has not yet been achieved much. Gallay-Wayne [7] proved the local stability of asymmetric Burgers vortices when $\lambda$ is sufficiently small. In Gallay-Wayne [6] the local stability with respect to three dimensional perturbations is obtained for $\lambda \in[0,1)$ when $|\alpha|$ is sufficiently small. In [14] it is proved that the asymmetric Burgers vortices are locally stable with respect to two dimensional perturbations when $\lambda \in\left[0, \frac{1}{2}\right)$ and $|\alpha|$ is sufficiently large. However it is still open whether or not the local stability of asymmetric Burgers vortices holds in general. In particular, when $\lambda \in\left[\frac{1}{2}, 1\right)$ we do not know whether the asymmetric Burgers vortices obtained in Theorem 1.2 are locally stable or not even in the case of sufficiently large $|\alpha|$. Finally, the global stability is not obtained so far in any asymmetric case $\lambda \in(0,1)$.

\section{REFERENCES}

[1] Burgers, J. M., A mathematical model illustrating the theory of turbulence. Adv. Appl. Mech., 1948, 171-199.

[2] Carlen, E. A. and Loss, M., Optimal smoothing and decay estimates for viscously damped conservation laws, with applications to the 2-D Navier-Stokes equation. Duke Math. J., 81, 1996, 135-157.

[3] Carpio, A., Asymptotic behavior for the vorticity equations in dimensions two and three. Commun. P. D. E., 19, 1994, 827-872.

[4] Engel, K.-J. and Nagel, R., One-Parameter semigroups for linear evolution equations. Graduate Texts in Mathematics. NewYork: Springer-Verlag, 2000.

[5] Gallay, Th. and Wayne, C. E., Global Stability of vortex solutions of the two dimensional Navier-Stokes equation. Comm. Math. Phys., 255(1), 2005, 97-129.

[6] Gallay, Th. and Wayne, C. E., Three-dimensional stability of Burgers vortices : the low Reynolds number case. Phys. D., 213, 2006, no. 2, 164-180.

[7] Gallay, Th. and Wayne, C. E., Existence and stability of asymmetric Burgers vortices. J. Math. Fluid Mech. 9, 2007, no. 2, 243-261. 
[8] Giga, Y. and Giga, M.-H., Nonlinear Partial Differential Equation, Self-similar solutions and asymptotic behavior; Kyoritsu: 1999 (in Japanese), English version to be published by Birkhäuser.

[9] Giga, Y. and Kambe, T., Large time behavior of the vorticity of two dimensional viscous flow and its application to vortex formation. Comm. Math. Phys., 117(1988), pp. 549-568.

[10] Kida, S. and Ohkitani, K., Spatiotemporal intermittency and instability of a forced turbulence. Phys. Fluids A, 4(5), 1992, 1018-1027.

[11] Lunardi, A., Analytic semigroups and optimal regularity in parabolic problems. Progress in Nonlinear Differential Equations and their Applications, 16. Birkh user Verlag, Basel, 1995. xviii+424 pp. ISBN: 3-7643-5172-1.

[12] Moffatt, H. K., Kida, S., and Ohkitani, K., Stretched vortices-the sinews of turbulence; largeReynolds-number asymptotics. J. Fluid Mech., 259, 1994, 241-264.

[13] Maekawa, Y., Spectral properties of the linearization at the Burgers vortex in the high rotation limit. to appear in J. Math. Fluid Mech.

[14] Maekawa, Y., On the existence of Burgers vortices for high Reynolds numbers. submitted.

[15] Prochazka, A. and Pullin, D. I., On the two-dimensional stability of the axisymmetric Burgers vortex. Phys. Fluids, 7(7), 1995, 1788-1790.

[16] Prochazka, A. and Pullin, D. I., Structure and stability of non-symmetric Burgers vortices. J. Fluid Mech., 363, 1998, 199-228.

[17] Robinson. A. C. and Saffman, P. G., Stability and Structure of stretched vortices. Stud. Appl. Math., 70(2), 1984, 163-181. 